\title{
Subspace intersection method of high-resolution bearing estimation in shallow ocean
}

\author{
Sondur Lakshmipathi, G.V. Anand* \\ Department of Electrical Communication Engineering, Indian Institute of Science, Bangalore 560 012, India
}

Received 25 February 2001; received in revised form 19 April 2004

\begin{abstract}
Plane-wave DOA estimation techniques yield biased estimates of source bearing angles in the ocean due to the multimode nature of acoustic propagation in an oceanic waveguide. Bearing estimation using matched field processing requires either a computationally expensive three-dimensional search or a priori knowledge of the source ranges and depths. A new subspace-based high-resolution bearing estimation technique which does not have any of these drawbacks is presented in this paper.
\end{abstract}

(C) 2004 Elsevier B.V. All rights reserved.

Keywords: Bearing estimation; High resolution; Normal modes; Shallow ocean

\section{Introduction}

Bearing estimation of acoustic sources is an important aspect of passive localization of targets in the ocean. It is known that conventional delay-and-sum beamforming yields biased estimates of the source bearing due to the multimode nature of acoustic propagation in the ocean $[2,6]$. For the same reason, other plane-wave direction-of-arrival (DOA) estimation techniques such as MUSIC [13], ESPRIT [12], and min-norm [9] algorithms also yield biased bearing estimates in the ocean. The bias increases as the number of propagating modes increases or as the bearing angle (with respect to the broadside direction of a linear horizontal array) increases. Unbiased bearing

\footnotetext{
* Corresponding author.

E-mail address: anandgv@ece.iisc.ernet.in (G.V. Anand).
}

estimates can be obtained using matched field processing techniques [16] such as the Bartlett processor or three-dimensional MUSIC. But these methods involve a computationally expensive three-dimensional search in the bearing-range-depth space unless prior estimates of range and depth are available.

In this paper, we present a new high-resolution method, called the subspace intersection method, for obtaining unbiased bearing estimates of multiple uncorrelated sources in a horizontally stratified ocean using a one-dimensional search without the prior knowledge of the source ranges and depths. The organization of the paper is as follows. The acoustic field structure in a horizontally stratified ocean is described in Section 2. The maximum likelihood (ML) and MUSIC estimation techniques are reviewed in Section 3, and the limitations of these techniques are discussed especially with reference to their computational 
complexity. The new subspace intersection method is described in Section 4. Salient features of the new bearing estimation technique are discussed in Section 5. A detailed performance analysis is carried out in Section 6 with the help of simulation results. Conclusions are presented in Section 7.

\section{Acoustic field at the array}

We model the ocean as a horizontally stratified water layer of constant depth $d$ overlying a horizontally stratified bottom. This model implies that the ocean is range-independent, i.e. variation of its acoustic properties in the horizontal direction is negligible in the range of interest. Let $J$ mutually uncorrelated narrowband sources of center frequency $f_{0}$ be located at depths $z_{j}$ and ranges $r_{j}(j=1, \ldots, J)$ with respect to the first element of a uniform linear horizontal array of $N$ narrowband sensors located at depth $z$ and having interelement spacing $\Delta$. Let the bearing angle of the $j$ th source with respect to the endfire direction of the array be denoted by $\theta_{j}$. The geometry of the problem is shown in Fig. 1.

Let the signal at the $n$th element of the array due to the $j$ th source be represented by

$$
\begin{aligned}
& s_{j n}(t)=p_{j n} \eta_{j}(t) \mathrm{e}^{\mathrm{i} 2 \pi f_{0} t}, \\
& \quad j=1, \ldots, J ; n=1, \ldots, N,
\end{aligned}
$$

where $\eta_{j}(t)$ is a slowly varying zero-mean random function that accounts for the random fluctuations of the source and the intervening medium, and the variance of $\eta_{j}(t)$ given by

$\sigma_{j}^{2}=E\left[\left|\eta_{j}(t)\right|^{2}\right]$

is a measure of the strength of the source. In Eq. (1), the signal amplitude $p_{j n}$ can be written as the sum of the discrete normal modes of the channel [1]

$p_{j n}=\sum_{m=1}^{M} b_{m j} \mathrm{e}^{\mathrm{i}(n-1) k_{m} \Delta \cos \theta_{j}}$,

where

$b_{m j}=\left(\frac{2 \pi}{k_{m} r_{j}}\right)^{1 / 2} \psi_{m}\left(z_{j}\right) \psi_{m}(z) \mathrm{e}^{-\alpha_{m} r_{j}-\mathrm{i}\left(k_{m} r_{j}-\pi / 4\right)}$

is the complex amplitude of the $m$ th normal mode at the first element of the array due to the $j$ th source, the

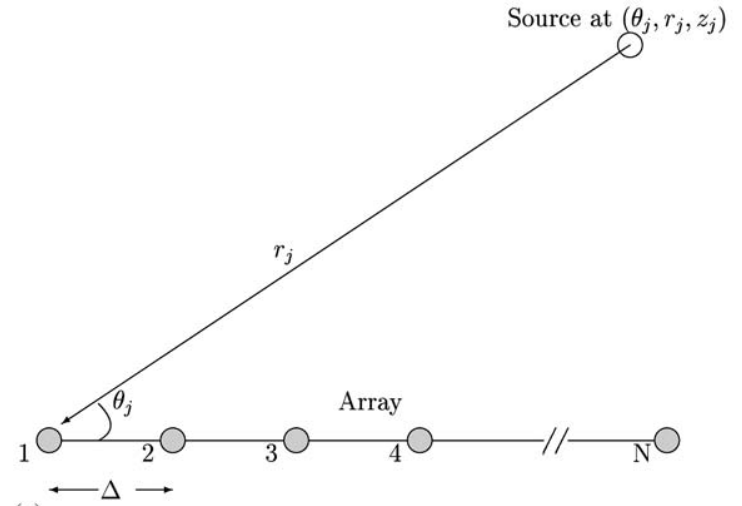

(a)

(b)

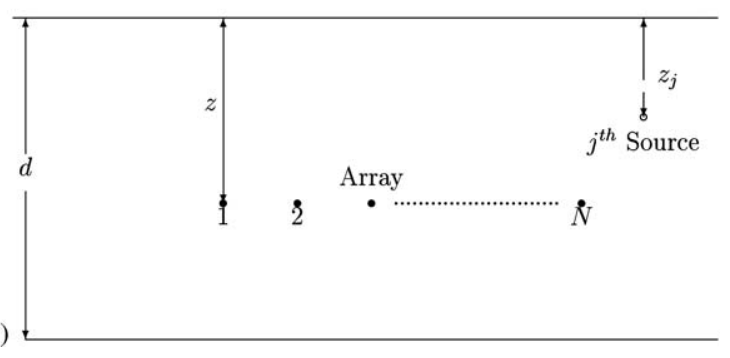

Fig. 1. Geometry of the source and receiver array, (a) top view (b) side view.

function $\psi_{m}(z)$ is the eigenfunction of the $m$ th normal mode of the oceanic waveguide, and the quantities $k_{m}$ and $\alpha_{m}$ are the corresponding wavenumber and attenuation coefficient, respectively. The output of the array of narrowband sensors can be expressed as the vector

$$
\begin{aligned}
\boldsymbol{y}(t) & =\left[y_{1}(t) \cdots y_{N}(t)\right]^{\mathrm{T}} \\
& =\boldsymbol{P}(\boldsymbol{X}) \boldsymbol{\eta}(t)+\boldsymbol{n}(t),
\end{aligned}
$$

where $\boldsymbol{\eta}(t)=\left[\eta_{1}(t) \cdots \eta_{J}(t)\right]^{\mathrm{T}}$ is the source signal vector, $\boldsymbol{n}(t)=\left[n_{1}(t) \cdots n_{J}(t)\right]^{\mathrm{T}}$ is the array noise vector, $\boldsymbol{X}=\left[\boldsymbol{x}_{1}^{\mathrm{T}} \cdots \boldsymbol{x}_{J}^{\mathrm{T}}\right]$,

$\boldsymbol{x}_{j}=\left[\begin{array}{lll}\theta_{j} & r_{j} & z_{j}\end{array}\right]^{\mathrm{T}}, \quad j=1, \ldots, J$

is the (unknown) position vector of the $j$ th source, and

$\boldsymbol{P}=\boldsymbol{P}(\boldsymbol{X})=\left[\boldsymbol{p}\left(\boldsymbol{x}_{1}\right) \cdots \boldsymbol{p}\left(\boldsymbol{x}_{J}\right)\right]$

is an $N \times J$ matrix whose columns

$\boldsymbol{p}\left(\boldsymbol{x}_{j}\right)=\left[p_{j 1} \cdots p_{j N}\right]^{\mathrm{T}}, \quad j=1, \ldots, J$

are the array signal amplitude vectors. The vectors $\boldsymbol{p}\left(\boldsymbol{x}_{j}\right)$ can, in turn, be expressed as

$\boldsymbol{p}\left(\boldsymbol{x}_{j}\right)=\boldsymbol{A}\left(\theta_{j}\right) \boldsymbol{b}\left(r_{j}, z_{j}\right), \quad j=1, \ldots, J$, 
where

$\boldsymbol{b}\left(r_{j}, z_{j}\right)=\left[b_{1 j} \cdots b_{M_{j}}\right]^{\mathrm{T}}, \quad j=1, \ldots, J$

are the mode amplitude vectors whose elements $b_{m j}$ are defined by Eq. (4), and

$\boldsymbol{A}(\theta)=\left[\boldsymbol{a}\left(k_{1} \cos \theta\right) \cdots \boldsymbol{a}\left(k_{M} \cos \theta\right)\right]$

is an $N \times M$ matrix whose columns are the steering vectors defined as

$$
\begin{gathered}
\boldsymbol{a}\left(k_{m} \cos \theta\right)=\left[1 \mathrm{e}^{\mathrm{i} k_{m} \Delta \cos \theta} \cdots \mathrm{e}^{\mathrm{i}(N-1) k_{m} \Delta \cos (\theta)}\right]^{\mathrm{T}}, \\
m=1, \ldots, M .
\end{gathered}
$$

\section{Review of bearing estimation techniques}

\subsection{ML estimator}

One method of estimating the source bearing angles $\theta_{1}, \ldots, \theta_{J}$ is to obtain the maximum likelihood estimate (MLE) of all the source coordinates $\boldsymbol{X}=\left[\begin{array}{lll}\boldsymbol{x}_{1}^{\mathrm{T}} & \boldsymbol{x}_{2}^{\mathrm{T}} \cdots \boldsymbol{x}_{J}^{\mathrm{T}}\end{array}\right]$. The MLE of $\boldsymbol{X}$, which is asymptotically efficient, maximizes the conditional log-likelihood function of $L$ observation vectors $\{\boldsymbol{y}(t), t=1, \ldots, L\}$ with respect to $\boldsymbol{X}$. An analytically tractable solution to this maximization problem is possible if the noise is Gaussian and spatially and temporally white, i.e.

$E\left[\boldsymbol{n}(t) \boldsymbol{n}^{\mathrm{H}}(t)\right]=\sigma^{2} \boldsymbol{I}$,

$E\left[\boldsymbol{n}(t) \boldsymbol{n}^{\mathrm{H}}(u)\right]=\mathbf{0} \quad$ for $t \neq u$.

Under these conditions, the conditional log-likelihood function is given by

$$
\begin{aligned}
\ell\left(\{\boldsymbol{y}(t)\}_{t=1}^{L} \mid \boldsymbol{\eta}(t)\right)= & \text { Constant }-N L \ln \sigma^{2} \\
& -\frac{1}{\sigma^{2}} \sum_{t=1}^{L}[\boldsymbol{y}(t)-\boldsymbol{P}(\boldsymbol{X}) \boldsymbol{\eta}(t)]^{\mathrm{H}} \\
& \times[\boldsymbol{y}(t)-\boldsymbol{P}(\boldsymbol{X}) \boldsymbol{\eta}(t)] .
\end{aligned}
$$

It can be shown [15] that the ML estimate of $\boldsymbol{X}$ minimizes the following cost function:

$$
C_{\mathrm{ML}}(\boldsymbol{X})=\operatorname{tr}\left([\boldsymbol{I}-\boldsymbol{\Pi}(\boldsymbol{X})]\left(\hat{\boldsymbol{C}}_{L}\right)\right),
$$

where

$\boldsymbol{\Pi}(\boldsymbol{X})=\boldsymbol{P}(\boldsymbol{X})\left[\boldsymbol{P}^{\mathrm{H}}(\boldsymbol{X}) \boldsymbol{P}(\boldsymbol{X})\right]^{-1} \boldsymbol{P}^{\mathrm{H}}(\boldsymbol{X})$,

$\hat{\boldsymbol{C}}_{L}=\frac{1}{L} \sum_{t=1}^{L} \boldsymbol{y}(t) \boldsymbol{y}^{\mathrm{H}}(t)$,

$\boldsymbol{I}$ is the $N \times N$ identity matrix, and $\operatorname{tr}($.$) denotes the$ trace of (.). The matrix $\hat{\boldsymbol{C}}_{L}$ is a consistent estimator of the data covariance matrix $\boldsymbol{C}$ defined as

$\boldsymbol{C}=E\left[\boldsymbol{y}(t) \boldsymbol{y}^{\mathrm{H}}(t)\right]$,

so that the sequence $\left\{\hat{\boldsymbol{C}}_{L}, L=1,2, \ldots\right\}$ converges in probability to $C$.

It is known [15] that the MLE is based on the assumption that the following conditions are satisfied:

- Condition $(\mathrm{C} 1): N>J$, and the signal vectors $\boldsymbol{p}(\boldsymbol{x})$ corresponding to every set of $(J+1)$ different values of $\boldsymbol{x}$ (i.e. every set of $(J+1)$ different source positions) are linearly independent. Satisfaction of this condition guarantees the uniqueness of the ML estimator.

- Condition(C2): Noise is spatially white, i.e. Eq. (14) is satisfied.

- Condition(C3): Noise is Gaussian and also temporally white, i.e. Eq. (15) is satisfied.

It is shown in Appendix A that a sufficient condition for the linear independence of every set of $(J+1)$ signal vectors with distinct bearing angles is that the sensor array satisfies the following constraints:

$N \geqslant M(J+1)$,

$\Delta<\pi / k_{1}=\lambda_{1} / 2$

where $\lambda_{1}$ is the wavelength of the first normal mode of the oceanic waveguide. Hence, unambiguous bearing estimation of $J$ sources is guaranteed if conditions (21) and (22) are satisfied.

A major drawback of the MLE is its computational complexity. Estimation of the positions of $J$ sources involves a search in a $3 J$-dimensional space.

\subsection{MUSIC estimator}

Multiple signal classification (MUSIC) is an estimation technique based on the eigendecomposition of 
the data covariance matrix $C$. We assume that the signal and noise are uncorrelated and that the noise is spatially white with variance $\sigma^{2}$. Using Eqs. (5)-(13), we can write

$$
\boldsymbol{C}=\left[\boldsymbol{p}\left(\boldsymbol{x}_{1}\right) \cdots \boldsymbol{p}\left(\boldsymbol{x}_{J}\right)\right] \boldsymbol{C}_{\mathrm{s}}\left[\boldsymbol{p}\left(\boldsymbol{x}_{1}\right) \cdots \boldsymbol{p}\left(\boldsymbol{x}_{J}\right)\right]^{\mathrm{H}}+\sigma^{2} \boldsymbol{I},
$$

where

$$
\boldsymbol{C}_{\mathrm{s}}=E\left[\boldsymbol{\eta}(t) \boldsymbol{\eta}^{\mathrm{H}}(t)\right]
$$

is the source covariance matrix. We assume that the sources are not fully correlated, so that the following condition is satisfied:

- Condition (C4): The matrix $\boldsymbol{C}_{\mathrm{s}}=E\left[\boldsymbol{\eta}(t) \boldsymbol{\eta}^{\mathrm{H}}(t)\right]$ is a full rank matrix, i.e. a matrix of rank $J$.

To proceed further with the development of the MUSIC algorithm we assume that condition (C1) is satisfied, i.e. signal vectors corresponding to every set of $(J+1)$ distinct source positions are linearly independent. It follows from this assumption that the matrix $\left[\boldsymbol{p}\left(\boldsymbol{x}_{1}\right) \cdots \boldsymbol{p}\left(\boldsymbol{x}_{J}\right)\right] \boldsymbol{C}_{\mathrm{s}}\left[\boldsymbol{p}\left(\boldsymbol{x}_{1}\right) \cdots \boldsymbol{p}\left(\boldsymbol{x}_{J}\right)\right]^{\mathrm{H}}$ in Eq. (23) is a matrix of rank $J$ whose unit-norm eigenvectors are equal to the $J$ unit-norm eigenvectors of $\boldsymbol{C}$ with the largest eigenvalues. Let these eigenvectors of $\boldsymbol{C}$ be denoted by $\boldsymbol{u}_{1}, \ldots, \boldsymbol{u}_{J}$, and let the remaining unit-norm eigenvectors of $\boldsymbol{C}$ be denoted by $\boldsymbol{u}_{J+1}, \ldots, \boldsymbol{u}_{N}$. The signal subspace $\mathscr{S}$ is defined as

$$
\mathscr{S}=\operatorname{span}\left\{\boldsymbol{u}_{1}, \ldots, \boldsymbol{u}_{J}\right\}=\operatorname{span}\left\{\boldsymbol{p}\left(\boldsymbol{x}_{1}\right), \ldots, \boldsymbol{p}\left(\boldsymbol{x}_{J}\right)\right\} .
$$

The noise subspace $\mathscr{N}$ defined as

$$
\mathscr{N}=\operatorname{span}\left\{\boldsymbol{u}_{J+1}, \ldots, \boldsymbol{u}_{N}\right\}
$$

is the orthogonal complement of $\mathscr{S}$. Let $T_{\mathrm{S}}=$ $\left\{\boldsymbol{x}_{1}, \ldots, \boldsymbol{x}_{J}\right\}$ be the set of source position vectors. It follows from the assumption of linear independence of the signal vectors $\boldsymbol{p}\left(\boldsymbol{x}_{1}\right), \ldots, \boldsymbol{p}\left(\boldsymbol{x}_{J}\right)$ that

$\boldsymbol{p}(\boldsymbol{x}) \in \mathscr{S}$ if and only if $\boldsymbol{x} \in T_{\mathrm{s}}$.

In other words, $\boldsymbol{p}(\boldsymbol{x})$ is orthogonal to the noise subspace $\mathscr{N}$ if and only if $\boldsymbol{x} \in T_{\mathrm{s}}$. Consequently, unique estimates of $\boldsymbol{x}_{1}, \ldots, \boldsymbol{x}_{J}$ are provided by the positions of the $J$ peaks of the ambiguity function

$$
B_{\text {MUSIC }}(\boldsymbol{x})=\left[\frac{\sum_{n=J+1}^{N}\left|\boldsymbol{p}^{\mathrm{H}}(\boldsymbol{x}) \boldsymbol{u}_{n}\right|^{2}}{\|\boldsymbol{p}(\boldsymbol{x})\|^{2}}\right]^{-1} .
$$

MUSIC requires a knowledge of the data covariance matrix $\boldsymbol{C}$. In practice, $\boldsymbol{C}$ is unknown, but it can be consistently estimated from the available data using Eq. (19). Accordingly, the unit-norm eigenvectors $\boldsymbol{u}_{n}$ in Eq. (28) are replaced by the unit-norm eigenvectors $\hat{\boldsymbol{u}}_{n}$ of the matrix $\hat{\boldsymbol{C}}_{L}$.

Conditions (C1) and (C2) required for MLE are also required for MUSIC. MUSIC estimator also requires the satisfaction of condition (C4). However, MUSIC does not require condition (C3), i.e. Gaussianity and temporal uncorrelatedness of noise is not required. This is a major advantage since acoustic noise in ocean is often non-Gaussian in character [11].

Another significant advantage of MUSIC over MLE is the reduction in computational complexity. MLE involves a search in a parameter space of dimension $3 J$, whereas MUSIC involves only a three-dimensional search. But even this three-dimensional search is quite demanding. It involves a lot of wasteful effort if one is interested in estimating only the source bearing angles.

\subsection{Plane-wave beamformers}

One method of reducing the computational complexity of the MUSIC algorithm is to approximate the vector $\boldsymbol{p}(\boldsymbol{x})$ in Eq. (28) as

$\boldsymbol{p}(\boldsymbol{x}) \simeq \boldsymbol{a}\left(k_{1} \cos \theta\right)$,

based on the approximation that the incident signal from each search direction is a plane wave. The choice of the wavenumber $k_{1}$ is based on the assumption that the first mode is the dominant mode in each incident signal. Eq. (28) reduces, under this approximation, to the well-known plane-wave MUSIC ambiguity function

$B_{\mathrm{PWM}}(\theta)=\left[\sum_{n=J+1}^{N}\left|\boldsymbol{a}^{\mathrm{H}}\left(k_{1} \cos \theta\right) \boldsymbol{u}_{n}\right|^{2}\right]^{-1}$.

The peaks of $B_{\mathrm{PWM}}(\theta)$, which are obtained through a one-dimensional search, yield biased estimates of the bearing angles $\theta_{j}$. An illustration of the biased bearing estimates provided by plane-wave MUSIC and the corresponding estimates by three-dimensional MUSIC beamformer [Eq. (28)] are given in Fig. 2. The ocean is modelled as a Pekeris channel [1] whose parameters are given in Section 6. A 40-element array with $\Delta=10 \mathrm{~m}$ is located at depth $z=50 \mathrm{~m}$. Three sources 




Fig. 2. Solid line: response of the plane-wave MUSIC beamformer for 3 sources at $\theta_{1}=10^{\circ}, \theta_{2}=45^{\circ}$ and $\theta_{3}=80^{\circ}$. Dash, dot, and dash-dot lines: response of the 3D-MUSIC beamformer for 3 sources at $\theta_{1}=10^{\circ}, \theta_{2}=45^{\circ}$ and $\theta_{3}=80^{\circ}$.

of frequency $50 \mathrm{~Hz}$ are located at range $10 \mathrm{~km}$, depth $20 \mathrm{~m}$ and bearing angles of $10^{\circ}, 45^{\circ}$ and $80^{\circ}$. It is seen from Fig. 2 (solid line) that the bias of the plane-wave MUSIC beamformer [Eq. (30)] is small only if the source is close to the broadside direction $\left(90^{\circ}\right)$, but the bias increases as the source moves away from the broadside direction. It can be shown that bearing estimates in the ocean obtained using a delay-and-sum beamformer exhibit a similar behavior $[2,6,7]$. The bias is also dependent on the source range and depth and on various ocean parameters. The response of the three-dimensional MUSIC beamformer has been obtained through three one-dimensional searches assuming prior knowledge of the range and depth of each source. Since the three estimates are obtained by three different one-dimensional searches, the curves are shown with dot, dash, and dot-dashed lines. The three-dimensional MUSIC yields unbiased bearing estimates, as expected.

\subsection{Limitations of ML and MUSIC estimators}

The ML and MUSIC estimators described in the preceding sections may be considered to belong to the broad class of matched field processors. In matched field processing (MFP) [16], the data vector $\boldsymbol{y}$ is matched with a set of replica signal vectors $s(\boldsymbol{\Phi})$, corresponding to a set of values of the parameter vector $\boldsymbol{\Phi}$, to find the best 'match'. The simplest and the most obvious way of matching the data vector with replica signal vectors is to use the Bartlett processor whose ambiguity function is defined as

$B_{\text {Bart }}(\boldsymbol{\Phi})=E\left[\left|\boldsymbol{s}^{\mathrm{H}}(\boldsymbol{\Phi}) \boldsymbol{y}\right|^{2}\right]=\boldsymbol{s}^{\mathrm{H}}(\boldsymbol{\Phi}) \boldsymbol{C} \boldsymbol{s}(\Phi)$,

where $\boldsymbol{C}=E\left[\boldsymbol{y} \boldsymbol{y}^{\mathrm{H}}\right]$ is the data covariance matrix. The Bartlett processor is essentially a linear processor which may be used for localization of a single source or for low-resolution localization of multiple sources. More sophisticated nonlinear processors are required for localization of multiple sources with a higher resolution. In general, the parameters required to compute the replica signal vectors may be divided into 3 categories. The first category consists of 'known' parameters which may be denoted by the vector $\boldsymbol{\Phi}^{(k)}$. The second category consists of parameters of 'interest' which are unknown parameters to be estimated, denoted by the vector $\boldsymbol{\Phi}^{(i)}$. The third category consists of 'nuisance' parameters which need not be estimated, denoted by the vector $\Phi^{(n)}$. Let $d^{(k)}, d^{(i)}$ and $d^{(n)}$ denote the dimensions of these parameter vectors. If there are no nuisance parameters, the search is conducted in a $d^{(i)}$-dimensional space to estimate the $d^{(i)}$ components of $\boldsymbol{\Phi}^{(i)}$. But, if nuisance parameters are present, $\boldsymbol{\Phi}^{(i)}$ and $\boldsymbol{\Phi}^{(n)}$ have to be estimated simultaneously even though we are not interested in estimating the components of $\boldsymbol{\Phi}^{(n)}$. Thus the dimension of the search space increases to $d^{(i)}+d^{(n)}$.

In the bearing estimation problem, all the channel parameters (viz., ocean depth, density and sound speed profile of water, and density and sound speed profile of ocean bottom), and the signal frequency are assumed to be known. The source powers $\left\{\sigma_{j}^{2}, j=1, \ldots, J\right\}$ are the unknown nuisance parameters, and the source positions $\left\{\theta_{j}, r_{j}, z_{j} ; j=1, \ldots, J\right\}$ are the unknown parameters of interest. In the case of the ML estimator, the problems of estimating the source powers and estimating the source positions can be decoupled from one another. Hence, for bearing estimation using MLE, the dimension of the search space is $3 J$. The enormous complexity of a high-dimensional search renders MLE unsuitable for multiple-source localization. MUSIC is representative of the class of MFP techniques in which the data vector is matched with the signal vectors from individual sources separately, and not with the total signal vector from $J$ sources. This mutual decoupling of sources is possible provided that the source covariance matrix $\boldsymbol{\Phi}=E\left[\boldsymbol{\eta}(t) \boldsymbol{\eta}^{\mathrm{H}}(t)\right]$ is a full-rank matrix. Under these conditions, $d^{(i)}=1, d^{(n)}=2$, and the 
dimension of the search space is $d^{(i)}+d^{(n)}=3$, irrespective of the number of sources. Therefore, the $J$ largest peaks of $B_{\text {MUSIC }}(\boldsymbol{x})$, where $\boldsymbol{x}$ is a three-dimensional vector, provide estimates of the positions of $J$ sources.

A common feature of all MFP based source localization algorithms is the need to know the channel parameters with sufficient accuracy. An incorrect choice of channel parameter values, known as environmental mismatch, leads to erroneous replica vector computations and hence to erroneous localization.

In the next section, we present the new subspaceintersection (SI) algorithm, which is a one-dimensional search technique for bearing angle estimation without involving the plane-wave approximation for the propagation of signals through the oceanic waveguide. The new method provides a significant reduction in computational complexity compared to the MUSIC estimator which involves a three-dimensional search. This simplification is achieved by isolating a feature of the signal vector that depends only on the source bearing angle. An additional advantage of the SI algorithm is that it does not require the knowledge of the channel parameters; a knowledge of the modal wavenumbers $\left\{k_{m}, m=1, \ldots, M\right\}$ is sufficient.

\section{Subspace intersection method}

\subsection{Modal subspace}

We consider a uniform horizontal linear array of $N$ sensors with intersensor spacing $\Delta$, satisfying conditions (21) and (22), i.e. $N \geqslant M(J+1)$ and $\Delta \leqslant \frac{1}{2} \lambda_{1}=$ $\pi / k_{1}$. A distinct steering vector $\boldsymbol{a}\left(k_{m} \cos \theta\right)$, defined by Eq. (13), is associated with each normal mode. We define the modal subspace $\mathscr{M}(\theta)$ as the span of all the modal steering vectors

$$
\begin{gathered}
\mathscr{M}(\theta)=\operatorname{span}\left\{\boldsymbol{a}\left(k_{1} \cos \theta\right), \ldots, \boldsymbol{a}\left(k_{M} \cos \theta\right)\right\}, \\
0 \leqslant \theta \leqslant \pi .
\end{gathered}
$$

For a given $\theta \neq \pi / 2$, the modal steering vectors are linearly independent, and hence the subspace $\mathscr{M}(\theta)$ is $M$-dimensional for $\theta \neq \pi / 2$.

Consider the modal subspaces $\mathscr{M}(\theta)$ and $\mathscr{M}\left(\theta^{\prime}\right)$ corresponding to two different steering angles $\theta$ and $\theta^{\prime}$. Inequalities (22) and (36) ensure that $\boldsymbol{a}\left(k_{m} \cos \theta\right) \neq \boldsymbol{a}\left(k_{m^{\prime}} \cos \theta^{\prime}\right) \quad \forall m \in\{1, \ldots, M\}$ and $\forall m^{\prime} \in\{1, \ldots, M\}$ if $\theta \neq \theta^{\prime}$. Hence the $2 M$ vectors $\left\{\boldsymbol{a}\left(k_{m} \cos \theta\right), \boldsymbol{a}\left(k_{m^{\prime}} \cos \theta^{\prime}\right), m, m^{\prime}=1, \ldots, M\right\}$ are linearly independent in view of inequality (21). Hence $\mathscr{M}(\theta) \neq \mathscr{M}\left(\theta^{\prime}\right)$ if $\theta \neq \theta^{\prime}$.

We shall now explore the relation between $\mathscr{M}(\theta)$ and the signal subspace $\mathscr{S}(\theta)$. Satisfaction of conditions (21) and (22) ensures that (see Section 3.1 and Appendix A) $J$ signal vectors $\boldsymbol{p}\left(\boldsymbol{x}_{1}\right), \ldots, \boldsymbol{p}\left(\boldsymbol{x}_{J}\right)$ corresponding to every set of $J$ sources with distinct bearing angles $\theta_{1}, \ldots, \theta_{J}$ are linearly independent. Hence, in accordance with Eq. (25), we can define the $J$-dimensional signal subspace $\mathscr{S}$ as

$$
\begin{aligned}
\mathscr{S} & =\operatorname{span}\left\{\boldsymbol{p}\left(\boldsymbol{x}_{1}\right), \ldots, \boldsymbol{p}\left(\boldsymbol{x}_{J}\right)\right\} \\
& =\operatorname{span}\left\{\boldsymbol{u}_{1}, \ldots, \boldsymbol{u}_{J}\right\} .
\end{aligned}
$$

It follows from Eqs. (10), (12) and (32) that

$\boldsymbol{p}\left(\boldsymbol{x}_{j}\right) \in \mathscr{M}\left(\theta_{j}\right), \quad j=1, \ldots, J$.

Hence, the subspaces $\mathscr{M}(\theta)$ and $\mathscr{S}$ intersect if $\theta \in\left\{\theta_{1}, \ldots, \theta_{J}\right\}$.

Let us now consider the structure of the signal vectors $\boldsymbol{p}\left(\boldsymbol{x}_{j}\right)$. We recall [see Eqs. (10)-(12)] that

$\boldsymbol{p}\left(\boldsymbol{x}_{j}\right)=\sum_{m=1}^{M} b_{m j} \boldsymbol{a}\left(k_{m} \cos \theta_{j}\right), \quad j=1, \ldots, J$.

The $m$ th mode amplitude $b_{m j}$ is zero only if either the $j$ th source or the sensor array is located at a node of the $m$ th normal mode. Hence, assuming that neither the source nor the array is at the ocean surface, we have $b_{1 j} \neq 0$. If we avoid locating the array at a node of any normal mode, at most one element of the set $\left\{b_{2 j}, \ldots, b_{M j}\right\}$ is zero. Finally, all the distinct elements of the set of $M(J+1)$ vectors $\left\{\boldsymbol{a}\left(k_{m} \cos \theta_{j}\right), \boldsymbol{a}\left(k_{m} \cos \theta\right) ; m=1, \ldots, M ; j=1, \ldots, J\right\}$ are linearly independent since $N \geqslant M(J+1)$. Recalling that the wavenumbers $k_{1}, \ldots, k_{M}$ are ordered as [1]

$k_{1}>k_{2}>\cdots>k_{M}$,

it follows that $\boldsymbol{p}\left(\boldsymbol{x}_{j}\right) \in \mathscr{M}(\theta)$ for $\theta \neq \theta_{j}$ if and only if the following conditions are satisfied:

$$
\begin{aligned}
\frac{k_{1}}{k_{2}} & =\frac{k_{2}}{k_{3}}=\cdots=\frac{k_{M-1}}{k_{M}} \\
& =\frac{\cos \theta}{\cos \theta_{j}}>1, \quad \text { and } \quad b_{M_{j}}=0 .
\end{aligned}
$$


Let us now consider a linear combination of two signal vectors which may be written as

$$
\begin{aligned}
\boldsymbol{p}\left(\boldsymbol{x}_{j}\right)+c \boldsymbol{p}\left(\boldsymbol{x}_{k}\right)= & \sum_{m=1}^{M}\left[b_{m j} \boldsymbol{a}\left(k_{m} \cos \theta_{j}\right)\right. \\
& \left.+c b_{m k} \boldsymbol{a}\left(k_{m} \cos \theta_{k}\right)\right],
\end{aligned}
$$

where $c$ is an arbitrary constant. Assuming that $\theta_{k}>\theta_{j}$, it follows that $\boldsymbol{p}\left(\boldsymbol{x}_{j}\right)+\boldsymbol{c} \boldsymbol{p}\left(\boldsymbol{x}_{k}\right) \in \mathscr{M}(\theta)$ for $\theta \notin\left\{\theta_{j}, \theta_{k}\right\}$ if and only if

$$
\begin{gathered}
\frac{k_{1}}{k_{2}}=\frac{k_{2}}{k_{3}}=\cdots=\frac{k_{M-1}}{k_{M}}=\frac{\cos \theta}{\cos \theta_{j}}=\frac{\cos \theta_{j}}{\cos \theta_{k}}, \\
c b_{(M-1) k}+b_{M j}=0, \quad b_{M k}=0 .
\end{gathered}
$$

Proceeding in a similar fashion it can be easily seen that, in a horizontally stratified ocean supporting 3 or more normal modes of propagation, a nontrivial linear combination of signal vectors belonging to the set $\left\{\boldsymbol{p}\left(\boldsymbol{x}_{1}\right), \ldots, \boldsymbol{p}\left(\boldsymbol{x}_{J}\right)\right\}$ can be an element of $\mathscr{M}(\theta)$ for $\theta \notin$ $\left\{\theta_{1}, \ldots, \theta_{J}\right\}$ only if the modal wavenumbers $k_{1}, \ldots, k_{M}$ form a geometric series, which may be considered to be an event of zero probability. It follows that the intersection of $\mathscr{S}$ and $\mathscr{M}(\theta)$ for $\theta \notin\left\{\theta_{1}, \ldots, \theta_{J}\right\}$ is an event of zero probability.

\subsection{Bearing estimation}

Let the $N \times(M+J)$ matrix $\boldsymbol{D}(\theta)$ be defined as

$\boldsymbol{D}(\theta)=\left[\frac{\boldsymbol{a}\left(k_{1} \cos \theta\right)}{\sqrt{N}} \cdots \frac{\boldsymbol{a}\left(k_{M} \cos \theta\right)}{\sqrt{N}} \boldsymbol{u}_{1} \cdots \boldsymbol{u}_{J}\right]$.

The first $M$ columns of $\boldsymbol{D}(\theta)$ are the (linearly independent) basis vectors of $\mathscr{M}(\theta)$ and the remaining $J$ columns are the orthonormal basis vectors of $\mathscr{S}$. The scaling factors $1 / \sqrt{N}$ appearing in the first $M$ columns of $\boldsymbol{D}(\theta)$ have been introduced for the sake of normalization. For convenience, we shall rewrite the matrix $\boldsymbol{D}(\theta)$ as

$\boldsymbol{D}(\theta)=\left[\boldsymbol{d}_{1}(\theta) \cdots \boldsymbol{d}_{P}(\theta)\right]$,

where

$$
P=M+J,
$$

$$
\boldsymbol{d}_{j}(\theta)= \begin{cases}\frac{\boldsymbol{a}\left(k_{j} \cos \theta\right)}{\sqrt{N}}, & 1 \leqslant j \leqslant M, \\ \boldsymbol{u}_{j-M}, & M+1 \leqslant j \leqslant P .\end{cases}
$$

Obviously, $\boldsymbol{d}_{j}(\theta)$ depends on $\theta$ only for $j \leqslant M$. Using QR decomposition [4], $\boldsymbol{D}(\theta)$ can be factorized as

$$
\boldsymbol{D}(\theta)=\boldsymbol{Q}(\theta) \boldsymbol{R}(\theta),
$$

where

$$
\boldsymbol{Q}(\theta)=\left[\boldsymbol{q}_{1}(\theta) \cdots \boldsymbol{q}_{P}(\theta)\right]
$$

is an $N \times P$ matrix whose columns $\boldsymbol{q}_{j}(\theta)$ are orthonormal vectors, and $\boldsymbol{R}(\theta)$ is a $P \times P$ upper triangular matrix with elements $r_{i j}(\theta)$. The columns of $\boldsymbol{D}(\theta)$ are related to the columns of $\boldsymbol{Q}(\theta)$ through the equations

$\boldsymbol{d}_{j}(\theta)=\sum_{i=1}^{j} r_{i j}(\theta) \boldsymbol{q}_{i}(\theta), \quad j=1, \ldots, P$,

and hence

$\boldsymbol{d}_{j}(\theta) \in \operatorname{span}\left\{\boldsymbol{q}_{1}(\theta), \ldots, \boldsymbol{q}_{j}(\theta)\right\}, \quad j=1, \ldots, P$.

The elements $r_{i j}(\theta)$ and the vectors $\boldsymbol{q}_{j}(\theta)$ can be determined recursively using the relations

$$
\begin{aligned}
& r_{11}(\theta)=\left\|\boldsymbol{d}_{1}(\theta)\right\|_{2}, \quad \boldsymbol{q}_{1}(\theta)=\frac{\boldsymbol{d}_{1}(\theta)}{r_{11}(\theta)}, \\
& r_{i j}(\theta)=\boldsymbol{q}_{i}^{\mathrm{H}}(\theta) \boldsymbol{d}_{j}(\theta), \quad 1 \leqslant i \leqslant j-1, \\
& \quad j=2, \ldots, P,
\end{aligned}
$$

$$
\begin{aligned}
r_{j j}(\theta) & =\left\|\boldsymbol{d}_{j}(\theta)-\sum_{i=1}^{j-1} r_{i j}(\theta) \boldsymbol{q}_{i}(\theta)\right\|_{2}, \\
j & =2, \ldots, P, \\
\boldsymbol{q}_{j}(\theta) & =\frac{\boldsymbol{d}_{j}(\theta)-\sum_{i=1}^{j-1} r_{i j}(\theta) \boldsymbol{q}_{i}(\theta)}{r_{j j}(\theta)}, \\
j & =2, \ldots, P,
\end{aligned}
$$

where $\|.\|_{2}$ denotes the Euclidean norm. A diagonal element $r_{j j}(\theta)$ of the matrix $\boldsymbol{R}(\theta)$ is zero if and only if $\boldsymbol{d}_{j}(\theta) \in \operatorname{span}\left\{\boldsymbol{d}_{1}(\theta), \ldots, \boldsymbol{d}_{j-1}(\theta)\right\}$. We shall exploit this property in the formulation of the bearing estimation algorithm.

We know that the subspaces $\mathscr{M}(\theta)=\operatorname{span}\left\{\boldsymbol{d}_{1}(\theta)\right.$, $\left.\ldots, \boldsymbol{d}_{M}(\theta)\right\}$ and $\mathscr{S}=\operatorname{span}\left\{\boldsymbol{d}_{M+1}, \ldots, \boldsymbol{d}_{P}\right\}$ intersect if and only if $\theta \in\left\{\theta_{1}, \ldots, \theta_{J}\right\}$. Hence $\theta \in\left\{\theta_{1}, \ldots, \theta_{J}\right\}$ if and only if one of the following conditions is satisfied:

$$
\begin{aligned}
& \boldsymbol{d}_{j} \in \operatorname{span}\left\{\boldsymbol{d}_{1}(\theta), \ldots, \boldsymbol{d}_{M}(\theta)\right\} \\
& \quad \text { for some } j \in\{M+1, \ldots, P\}
\end{aligned}
$$


or

$\boldsymbol{d}_{P} \in \operatorname{span}\left\{\boldsymbol{d}_{1}(\theta), \ldots, \boldsymbol{d}_{P-1}(\theta)\right\}$.

We can combine conditions (52) and (53) to obtain the following result:

$\boldsymbol{d}_{j} \in \operatorname{span}\left\{\boldsymbol{d}_{1}(\theta), \ldots, \boldsymbol{d}_{j-1}(\theta)\right\}$

for some $j \in\{M+1, \ldots, P\}$

if and only if $\theta \in\left\{\theta_{1}, \ldots, \theta_{J}\right\}$.

Since $r_{j j}(\theta)=0$ if and only if $\boldsymbol{d}_{j}(\theta) \in \operatorname{span}\left\{\boldsymbol{d}_{1}(\theta)\right.$, $\left.\ldots, \boldsymbol{d}_{j-1}(\theta)\right\}$, it follows that $r_{j j}(\theta)=0$ for some $j \in\{M+1, \ldots, P\}$ if and only if $\theta \in\left\{\theta_{1}, \ldots, \theta_{J}\right\}$. Hence the function $\left\{\min _{M+1 \leqslant j \leqslant P}\left|r_{j j}(\theta)\right|\right\}$ vanishes at $\theta=\theta_{1}, \theta_{2}, \ldots, \theta_{J}$.

The subspace-intersection (SI) algorithm for bearing estimation may be described as follows:

(1) Estimate the covariance matrix $\boldsymbol{C}$ from the array data vector $\boldsymbol{y}(t)$ by averaging $\boldsymbol{y}(t) \boldsymbol{y}^{\mathrm{H}}(t)$ over $L$ snapshots as in Eq. (19). Let the estimated covariance matrix be denoted by $\hat{\boldsymbol{C}}$.

(2) Determine the eigenvectors of $\hat{\boldsymbol{C}}$. Divide the eigenvectors into two groups, viz. the signal eigenvectors $\hat{\boldsymbol{u}}_{1}, \ldots, \hat{\boldsymbol{u}}_{J}$ and the noise eigenvectors $\hat{\boldsymbol{u}}_{J+1}, \ldots, \hat{\boldsymbol{u}}_{N}$.

(3) Form the matrix $\hat{\boldsymbol{D}}(\theta)$ by appending the columns $\hat{\boldsymbol{u}}_{1}, \ldots, \hat{\boldsymbol{u}}_{J}$ to the columns $\boldsymbol{a}\left(k_{1} \cos \theta\right) / \sqrt{N}, \ldots$, $\boldsymbol{a}\left(k_{M} \cos \theta\right) / \sqrt{N}$.

(4) Do the QR factorization of $\hat{\boldsymbol{D}}(\theta)$ as $\hat{\boldsymbol{D}}(\theta)=$ $\hat{\boldsymbol{Q}}(\theta) \hat{\boldsymbol{R}}(\theta)$. Let $\hat{r}_{j j}(\theta)$ denote the diagonal elements of the upper triangular matrix $\hat{\boldsymbol{R}}(\theta)$.

(5) Compute the function

$B_{\mathrm{SI}}(\theta)=\left[\min _{M+1 \leqslant j \leqslant P} \hat{r}_{j j}(\theta)\right]^{-1}$.

The location of the peaks of $B_{\mathrm{SI}}(\theta)$ provides estimates of the source bearing angles.

\section{Discussion}

Before presenting numerical results to illustrate the performance of the SI algorithm, we shall discuss its salient qualitative features. The conditions $N \geqslant M(J+1)$ and $\Delta \leqslant \lambda_{1} / 2$ are incorporated in the SI algorithm to ensure that the signal vectors are linearly independent and that the intersection of $\mathscr{S}$ and
$\mathscr{M}(\theta)$ is extremely unlikely when $\theta \neq\left\{\theta_{1}, \ldots, \theta_{J}\right\}$. A necessary condition for the columns of $\boldsymbol{D}(\theta)$ to be linearly independent when $\theta \notin\left\{\theta_{1}, \ldots, \theta_{J}\right\}$ is

$N \geqslant M+J$.

Usually, the satisfaction of this necessary condition is adequate for achieving unambiguous bearing estimation as borne out by the simulation results presented in Section 6. Simulation results also indicate that values of $\Delta$ well in excess of $\lambda_{1} / 2$ may be used without introducing any ambiguity. This tolerance to larger values of $\Delta$ is due to the multimodal nature of the signal and the noncoincidence of the ambiguity directions for different normal modes. The problem of determination of the minimal sufficient conditions on $N$ and $\Delta$ for unambiguous bearing estimation still remains unresolved.

The resolution provided by the SI algorithm is determined by the sharpness of the peaks of the function $B_{\mathrm{SI}}(\theta)$. In general, the resolution would depend on the parameters $N, \delta$, the array depth, the source position, and the number of snapshots used to estimate the covariance matrix $\boldsymbol{C}$. The determination of an analytical expression for resolution appears to be a difficult task. But we can get some useful insights by considering a definition of resolution that is not specific to the SI algorithm, but one that may be applied to any bearing estimation algorithm. We note that bearing estimation is performed by identifying the values of $\theta$ at which $\mathscr{S}$ and $\mathscr{M}(\theta)$ intersect. If a source is located at $\left(\theta, r_{j}, z_{j}\right)$, the unit vector that is common to $\mathscr{S}$ and $\mathscr{M}(\theta)$ is given by [see Eqs. (33)-(35)]

$\boldsymbol{e}(\theta)=\frac{\sum_{m=1}^{M} b_{m j} \boldsymbol{a}\left(k_{m} \cos \theta\right)}{\left\|\sum_{m=1}^{M} b_{m j} \boldsymbol{a}\left(k_{m} \cos \theta\right)\right\|_{2}}$,

where $b_{m j}$ is the complex mode amplitude that depends on the range $r_{j}$ and depth $z_{j}$ [see Eq. (14)]. Hence, we define

$Q(\theta)=\left\|\frac{\mathrm{d}(\theta)}{\mathrm{d} \theta}\right\|_{2}$

as a measure of resolution in the direction $\theta$. It is shown in Appendix B that for $N \gg 1$,

$Q(\theta) \cong \frac{1}{\sqrt{3}} N \Delta\left\{\frac{\sum_{m=1}^{M} k_{m}^{2}\left|b_{m j}\right|^{2}}{\sum_{m=1}^{M}\left|b_{m j}\right|^{2}}\right\}^{1 / 2}|\sin \theta|$. 
Thus, the resolution depends not only on the source direction but also on the range and depth. The resolution may be improved either by increasing the number of sensors $N$ or by increasing intersensor spacing $\Delta$. However, it must be remembered that values of $\Delta$ greater than $\lambda_{1} / 2$ may lead to ambiguity due to the appearance of false peaks in the function $B_{\mathrm{SI}}(\theta)$. Eq. (59) also indicates that resolution declines as one approaches the endfire direction $(\theta=0$ or $\pi)$.

It is interesting to note that the expression for $Q(\theta)$ in the last equation depends on a weighted root-mean-square value of the modal wavenumbers, the weights being proportional to the modal amplitudes. Since the wavenumbers are ordered as $k_{1}>k_{2}>\cdots>k_{M}$, it follows that resolution is higher for those source/array positions for which the lower order modes are predominant.

The bearing estimation accuracy of the SI algorithm depends on the accuracy with which the signal eigenvectors $\boldsymbol{u}_{1}, \ldots, \boldsymbol{u}_{J}$ are estimated. Errors in the estimation of $\left\{\boldsymbol{u}_{j} ; j=1, \ldots, J\right\}$ are caused by the error in estimating the covariance matrix $\boldsymbol{C}$ from a finite number of samples or snapshots of the data vector. The covariance matrix estimation error can be reduced by increasing the number of snapshots $L$; but increases in processing delay and computational complexity due to increasing $L$ impose an upper limit on the value of $L$. For a given $L$, the covariance matrix estimation error increases as the signal-to-noise ratio is reduced [16], leading to a progressive degradation of the SI processor performance.

The outstanding feature of the SI technique is that it retains the computational simplicity of one-dimensional search without invoking the planar wavefront assumption or requiring a priori knowledge of source ranges and depths. Another advantage of the SI technique over the MFP techniques is that the former requires less information about the environment. MFP requires the knowledge of a host of ocean acoustic parameters such as the density, sound speed and sound absorption profiles of the sea water and sediment, and roughness of the sea surface. The values of these parameters, some of which are time varying, are often not known with sufficient accuracy, and their estimation is quite a difficult task. On the other hand, the SI algorithm requires only the knowledge of modal wavenumbers $\left\{k_{m}, m=1, \ldots, M\right\}$, and several methods of estimating the wavenumbers accurately are known $[3,8,14]$. Hence, the SI technique is much less susceptible to environmental mismatch than MFP techniques. The SI technique shares with MUSIC the capabilities of high-resolution and multiple-source bearing estimation. Like MUSIC, the SI technique too requires prior knowledge of the number of sources for reliable estimation of the signal subspace.

\section{Simulation results}

Simulation results on the performance of the SI algorithm are presented in this section for two ocean models. The first model is the ideal Pekeris channel [1] consisting of a homogeneous water layer of constant depth overlying a homogeneous fluid half-space. The channel parameters are: water depth $=d=100 \mathrm{~m}$, sound speed in water $=c=1500 \mathrm{~m} / \mathrm{s}$, sound speed in bottom $=c_{\mathrm{b}}=1700 \mathrm{~m} / \mathrm{s}$, ratio of density of bottom to density of water $=\rho_{\mathrm{b}} / \rho=1.5$, attenuation in bottom $=\delta=0.5 \mathrm{~dB} / \lambda$. The second model corresponds to a site in the Gulf of Mexico [5] with a mean water depth $d=115 \mathrm{~m}$, and a range-independent sound-speed profile in water as shown in Fig. 3. In this model, both the surface and bottom of the ocean are rough with an rms height of $0.1 \mathrm{~m}$, and the bottom has two sediment layers of thicknesses $d_{1}=d_{2}=50 \mathrm{~m}$, sound speeds $c_{\mathrm{b}_{1}}=1780 \mathrm{~m} / \mathrm{s}$, and $c_{\mathrm{b}_{2}}=1800 \mathrm{~m} / \mathrm{s}$, and density ratios $\rho_{\mathrm{b}_{1}} / \rho=1.7$ and $\rho_{\mathrm{b}_{2}} / \rho=1.9$, overlying a rigid half-space. The acoustic field in the Pekeris channel can be computed easily [1], and simulation results for this channel are presented in considerable detail to assess the effect of variation of several parameters, viz. the number of elements in the array, interelement spacing, number of modes propagating in the channel, signal-to-noise ratio, and number of snapshots. The effect of errors in the assumed values of the wavenumbers on the SI processor performance is also investigated. Acoustic field computations in the Gulf of Mexico channel are carried out using the Kraken normal mode program [5]. Results for this channel are presented to demonstrate that the SI algorithm is equally effective in estimating source bearing in a realistic environment.

The output of each sensor in the array is the sum of signals received from $J$ noncoherent sources and an independent zero-mean spatially white Gaussian 


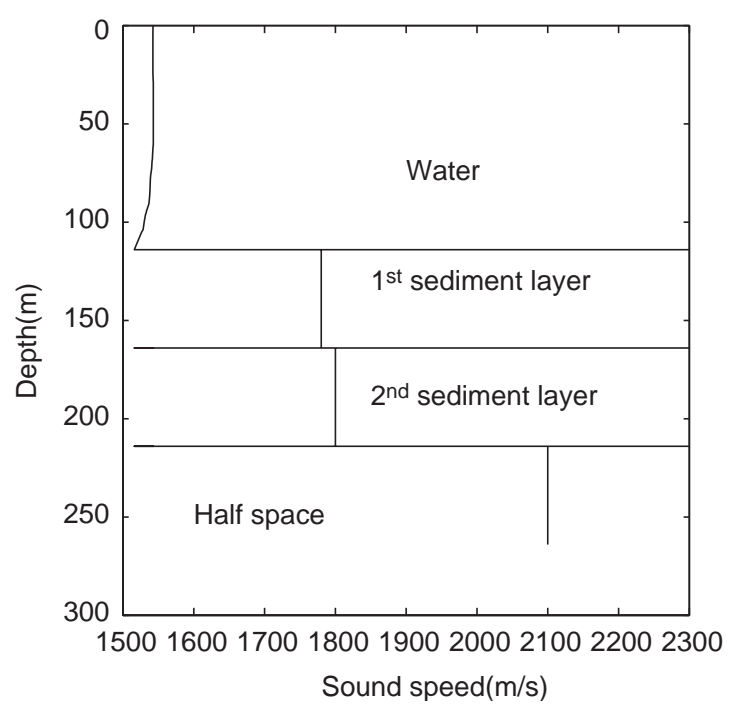

Fig. 3. Sound speed profile in the Gulf of Mexico channel. 1st sediment layer: $c_{\mathrm{b}}=1780 \mathrm{~m} / \mathrm{s}, \rho_{\mathrm{b}} / \rho=1.7, \delta=0.24 \mathrm{~dB} / \lambda$. 2nd sediment layer: $c_{\mathrm{b}}=1800 \mathrm{~m} / \mathrm{s}, \rho_{\mathrm{b}} / \rho=1.9, \delta=0.32 \mathrm{~dB} / \lambda$. Half space: $c_{\mathrm{b}}=2100 \mathrm{~m} / \mathrm{s}, \rho_{\mathrm{b}} / \rho=2.12$.

noise. The $l$ th sample of the simulated output of the $q$ th element of the array is given by

$y_{q}(l)=\sum_{j=1}^{J} p_{j q} \eta_{j}(l) \mathrm{e}^{\mathrm{i} \phi_{j}(l)}+n_{q}(l)$,

where $\left\{n_{q}(l), q=1, \ldots, N ; l=1, \ldots, L\right\}$ are independent sample values of a circular complex zero-mean Gaussian random variable with variance $\sigma^{2}$. Independent samples of uniformly distributed random phases $\phi_{j}(l)$ are included to render the sources noncoherent. The quantities $\left\{p_{j q}, j=1, \ldots, J ; q=1, \ldots, N\right\}$ are computed from Eqs. (3) and (4). The signal-to-noise ratio (SNR) is defined as the logarithmic ratio of the total signal power at the array to the total noise power, i.e.,

$\mathrm{SNR}=10 \log _{10} \frac{\sum_{q=1}^{N} E\left[\left|\sum_{j=1}^{J} p_{j q} \eta_{j}(l)\right|^{2}\right]}{N E\left[\left|n_{q}(l)\right|^{2}\right]}$.

Since the sources are noncoherent, Eq. (61) reduces to

$\mathrm{SNR}=10 \log _{10} \frac{\sum_{q=1}^{N} \sum_{j=1}^{J} \sigma_{j}^{2}\left|p_{j q}\right|^{2}}{N \sigma^{2}}$

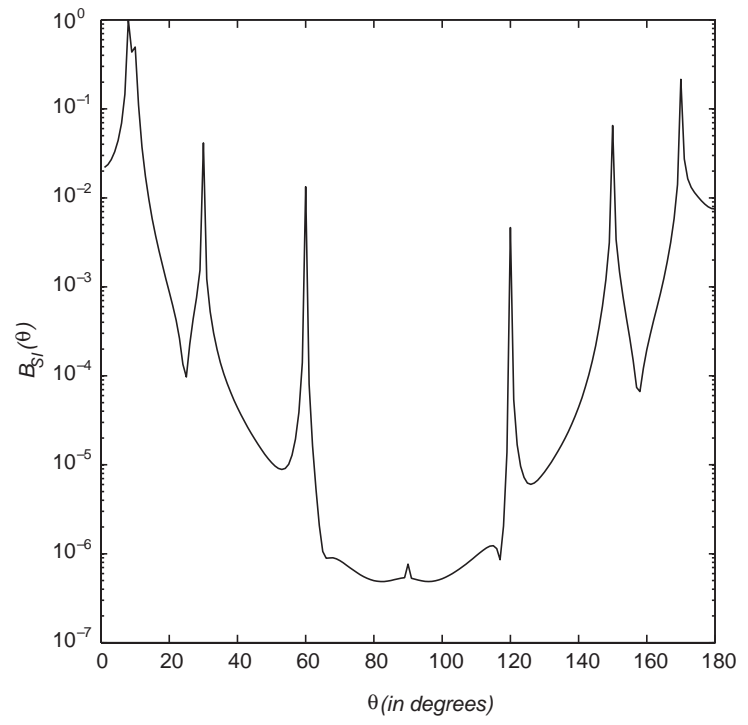

Fig. 4. SI processor response for 6 sources at $10^{\circ}, 30^{\circ}, 60^{\circ}, 120^{\circ}$, $150^{\circ}$, and $170^{\circ}$ in a noiseless Pekeris channel. $f=50 \mathrm{~Hz}, N=9$, $\Delta=\lambda / 2$.

The elements of the estimated data correlation matrix $\hat{\boldsymbol{C}}$ are given by (see Eq. (19))

$$
\begin{aligned}
\hat{c}_{n q} & =\frac{1}{L} \sum_{l=1}^{L} y_{n}(l) y_{q}^{*}(l), \quad n=1, \ldots, N, \\
q & =1, \ldots, N .
\end{aligned}
$$

In all the simulations except those in Figs. 4-6 and 13, the matrix $\hat{\boldsymbol{C}}$ was computed from 200 snapshots $(L=200)$ of the data vector, and the bearing estimates were obtained by averaging over 100 simulations. Fig. 4 shows the SI processor response $B_{\mathrm{SI}}(\theta)$ in the Pekeris channel when 6 sources at $10^{\circ}, 30^{\circ}, 60^{\circ}$, $120^{\circ}, 150^{\circ}$ and $170^{\circ}$ bearing are present in a noiseless environment. All the sources are at $10 \mathrm{~km}$ range and $51 \mathrm{~m}$ depth. The signal frequency $f$ is $50 \mathrm{~Hz}$, and the number of modes $M$ at this frequency is 3 . The number of elements $N$ in the array is 9 , which is the minimum number required to satisfy the necessary condition $N \geqslant M+J$. The interelement distance is $\lambda / 2$, i.e. $15 \mathrm{~m}$. The response function has well-defined peaks whose positions match very well with the source directions. In a noisy environment, the same performance is achieved asymptotically as $L \rightarrow \infty$. Fig. 5 shows the asymptotic response in the Gulf of Mexico 


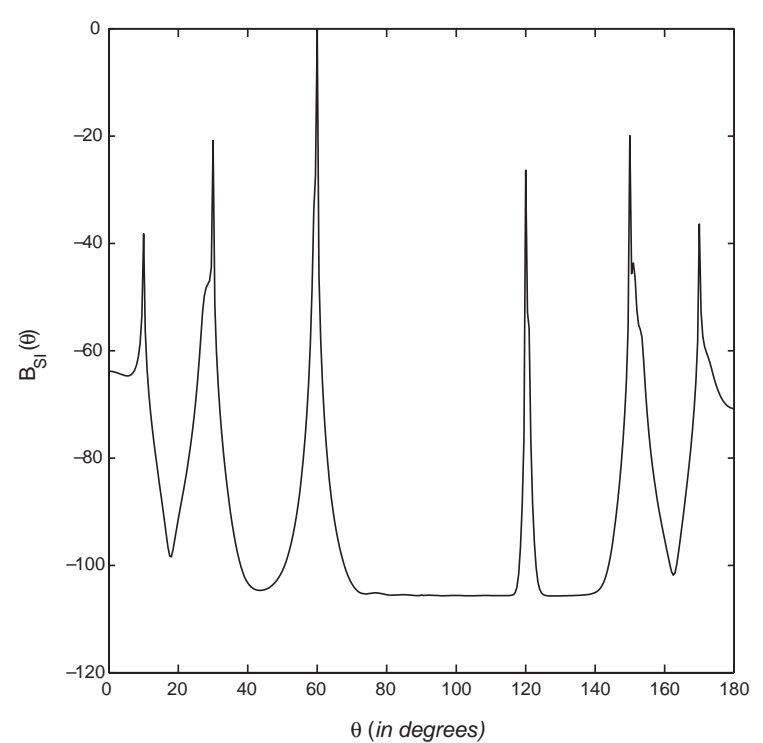

Fig. 5. Asymptotic response of SI processor for 6 sources at $10^{\circ}$, $30^{\circ}, 60^{\circ}, 120^{\circ}, 150^{\circ}$, and $170^{\circ}$ in the Gulf of Mexico channel. $f=50 \mathrm{~Hz}, N=30, \Delta=\lambda / 2$.

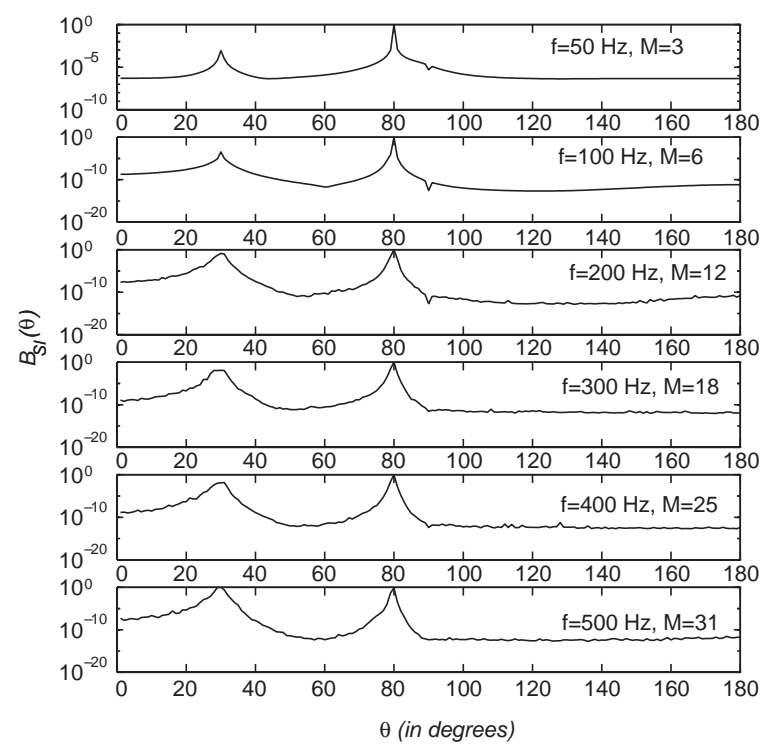

Fig. 6. Asymptotic performance of the SI processor at different frequencies for 2 sources at $30^{\circ}$ and $80^{\circ} . N=M+2$ and $\Delta=\lambda / 2$ in each case.

channel for the same source locations and for $N=30$. It is seen that the performance of the SI processor in the Gulf of Mexico channel is qualitatively similar to that in the Pekeris channel.

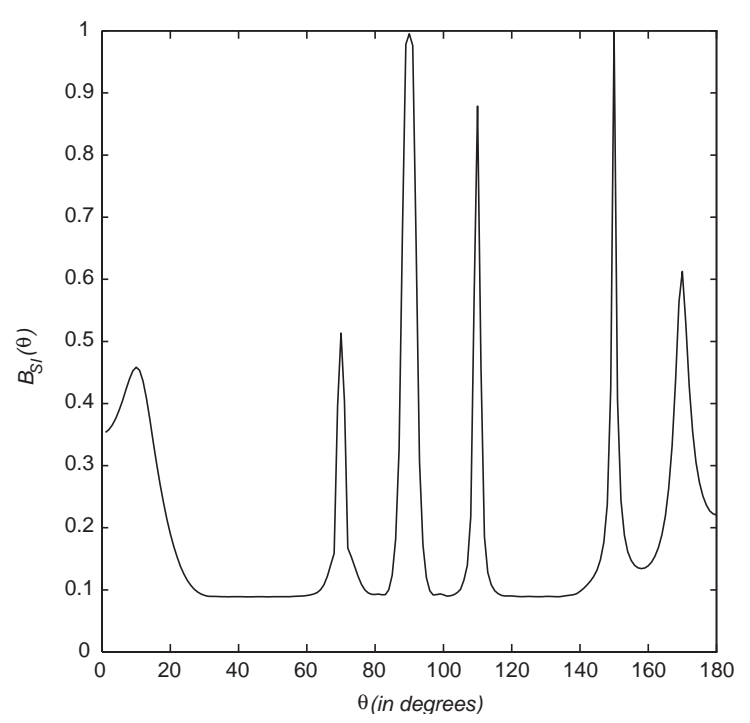

Fig. 7. Finite-data performance of SI processor in the Pekeris channel at $20 \mathrm{~dB}$ SNR with 200 snapshots and 100 Monte Carlo simulations. Six sources are located at $10^{\circ}, 70^{\circ}, 90^{\circ}, 110^{\circ}, 150^{\circ}$, and $170^{\circ} . f=50 \mathrm{~Hz}, N=30, \Delta=\lambda / 2$.

The asymptotic performance of the SI processor at different frequencies for 2 sources at $30^{\circ}$ and $80^{\circ}$ bearings, is shown in Fig. 6. As the frequency increases, the number of modes $M$ also increases, and the number of sensors $N$ is chosen so that $N=M+J$. Figs. 4 and 6 show that in a channel that supports $M$ normal modes, it is possible to achieve unbiased bearing estimation of $J$ sources using an array of $M+J$ sensors if $\boldsymbol{C}$ is known exactly. But, if $\boldsymbol{C}$ is estimated from finite data, a larger value of $N$ has to be used to overcome the correlation matrix estimation error.

Fig. 7 shows the plot of $B_{\mathrm{SI}}(\theta)$ for the finite data case, obtained using an array of 30 sensors with $\lambda / 2$-spacing. The SNR is $20 \mathrm{~dB}$, and results of 100 simulations of 200 snapshots each have been averaged to generate the plot in Fig. 7. The source bearing angles are $10^{\circ}, 70^{\circ}, 90^{\circ}, 110^{\circ}, 150^{\circ}$ and $170^{\circ}$. All the other parameters have the same values as before. Unbiased, high-resolution bearing estimation has been achieved once again. Fig. 8 shows the result of repeating an identical experiment in the Gulf of Mexico channel. It is seen once again that the performance of the SI processor in the realistic environment of the Gulf of Mexico channel is similar to that in the idealized Pekeris channel. 


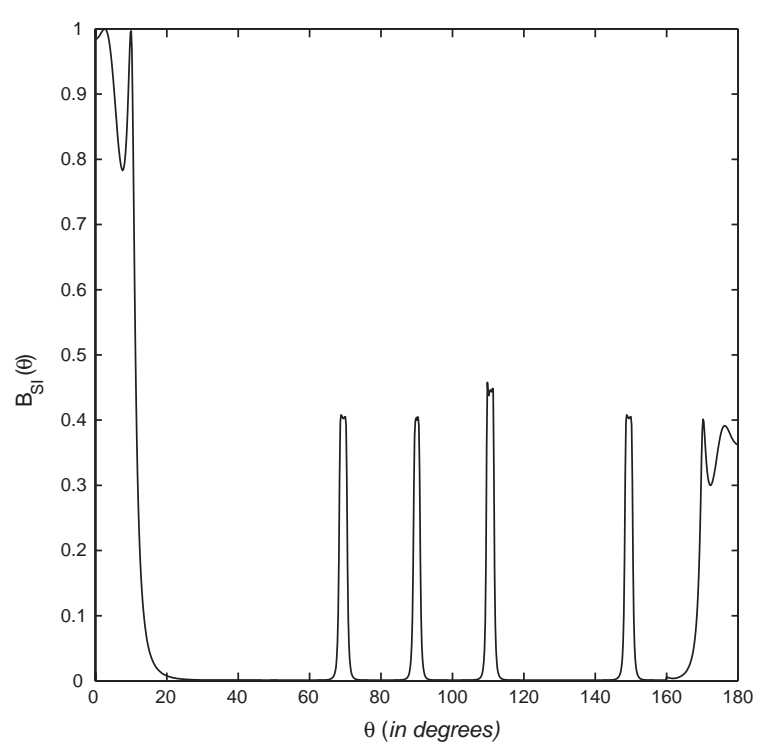

Fig. 8. Finite-data performance of SI processor in the Gulf of Mexico channel at $20 \mathrm{~dB}$ SNR with 200 snapshots and 100 Monte Carlo simulations. Six sources are located at $10^{\circ}, 70^{\circ}, 90^{\circ}, 110^{\circ}$, $150^{\circ}$, and $170^{\circ} \cdot f=50 \mathrm{~Hz}, N=30, \Delta=\lambda / 2$.

Having established the ability of the SI processor to achieve multiple-source bearing estimation in a horizontally stratified range-independent ocean, we shall now study the finite-data performance in greater detail. For the sake of computational simplicity, we shall henceforth confine our attention to Pekeris channel with one or two sources only. Values of $f, N, \Delta$, SNR and $L$ are the same as those in Fig. 7, unless otherwise stated.

Fig. 9 shows the bearing estimation of 2 sources at $10^{\circ}$ and $80^{\circ}$ for two different range-depth combinations viz. $r_{1}=r_{2}=3 \mathrm{~km}, z_{1}=z_{2}=21 \mathrm{~m}$ (solid line) and $r_{1}=r_{2}=10 \mathrm{~km}, z_{1}=z_{2}=51 \mathrm{~m}$ (broken line). Results in Fig. 9 as well as many other simulations carried out prove conclusively that bearing estimation can be done effectively for all source ranges and depths, though the heights of the main peaks of $B_{\mathrm{SI}}(\theta)$ vary in an apparently irregular manner.

The response functions of the SI processor and the plane-wave MUSIC processor are compared in Fig. 10 for 2 sources at $10^{\circ}$ and $80^{\circ}$. As expected, plane-wave MUSIC yields a biased bearing estimate of the source at $10^{\circ}$. However, for the near-broadside $\left(80^{\circ}\right)$ source, plane-wave MUSIC shows no bias and provides



Fig. 9. Finite-data performance of SI processor for 2 sources at $10^{\circ}$ and $80^{\circ}$ with different range-depth combinations. SNR $=20 \mathrm{~dB}$, $f=50 \mathrm{~Hz}, N=30, \Delta=\lambda / 2$. Solid line: $r_{1}=r_{2}=3 \mathrm{~km} ; z_{1}=z_{2}=21 \mathrm{~m}$. Dash-dot line: $r_{1}=r_{2}=10 \mathrm{~km} ; z_{1}=z_{2}=51 \mathrm{~m}$.



Fig. 10. Comparison of finite-data performance of SI and plane-wave MUSIC processors for 2 sources at $10^{\circ}$ and $80^{\circ}$. $\mathrm{SNR}=20 \mathrm{~dB}, f=50 \mathrm{~Hz}, N=30, \Delta=\lambda / 2$. Solid line: SI. Dashdot line: plane-wave MUSIC. SNR $=20 \mathrm{~dB}, f=50 \mathrm{~Hz}, N=30$. 

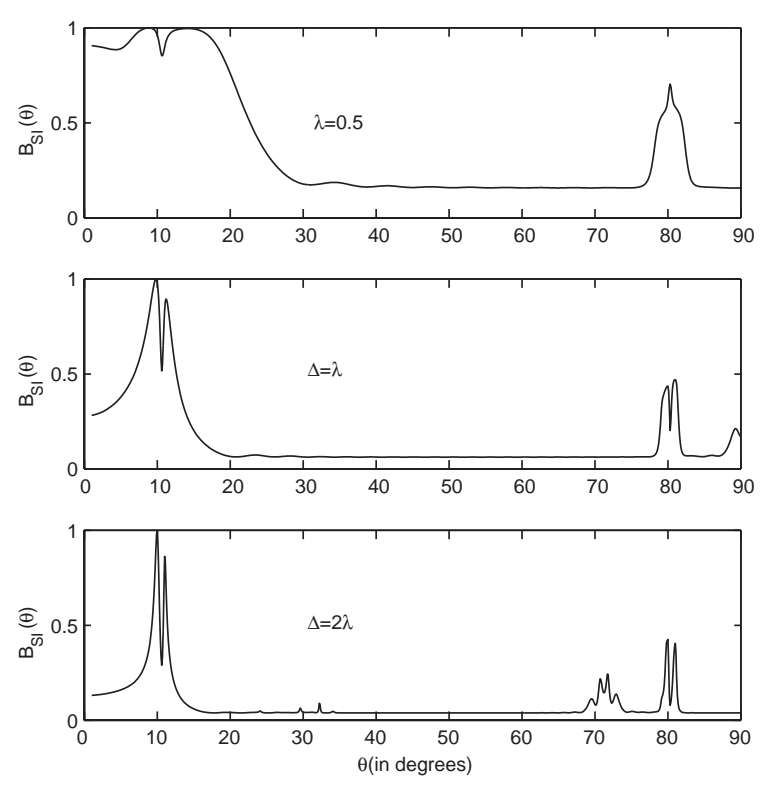

Fig. 11. Enhancement of the resolution of the SI processor by increasing the spacing $\Delta$ between sensors. The sources are located at $10^{\circ}, 11^{\circ}, 80^{\circ}$ and $81^{\circ} . \Delta=0.5 \lambda, \lambda$, and $2 \lambda$.

a higher resolution than the SI processor. The SI processor localizes both sources equally well.

The enhancement of the resolution of the SI processor by increasing the spacing $\Delta$ between sensors is illustrated in Fig. 11. The sources are located at $10^{\circ}$, $11^{\circ}, 80^{\circ}$ and $81^{\circ} . B_{\mathrm{SI}}(\theta)$ is shown for different values of $\Delta$, viz. $\Delta=0.5 \lambda, \lambda$, and $2 \lambda$. The simulation parameters are 30 sensors, $\mathrm{SNR}=20 \mathrm{~dB}, 100$ simulations of 200 snapshots each. For $\Delta=2 \lambda$ all the sources are well resolved except for some false peaks around $70^{\circ}$. It is noteworthy that unambiguous localization with high resolution can be achieved by choosing a sufficiently high value of $\Delta$ that is well above $\lambda / 2$. However, as $\Delta$ is increased, more and more ripples (sidelobes) appear in the response function $B_{\mathrm{SI}}(\theta)$. At larger values of $\Delta$ or lower SNR, the amplitude of a sidelobe, may become comparable to one of the main peaks.

Another way of improving resolution is to increase the number of sensors $N$. Plots of $B_{\mathrm{SI}}(\theta)$ for different values of $N$ are shown in Fig. 12 for sources at $10^{\circ}, 11^{\circ}, 80^{\circ}$ and $81^{\circ}$. The SNR is $0 \mathrm{~dB}$ and the interelement distance $\Delta$ is equal to $\lambda / 2$. It is seen that a good resolution can be achieved at $80^{\circ}$ with a 30 -sensor array, but 50 sensors are required to achieve
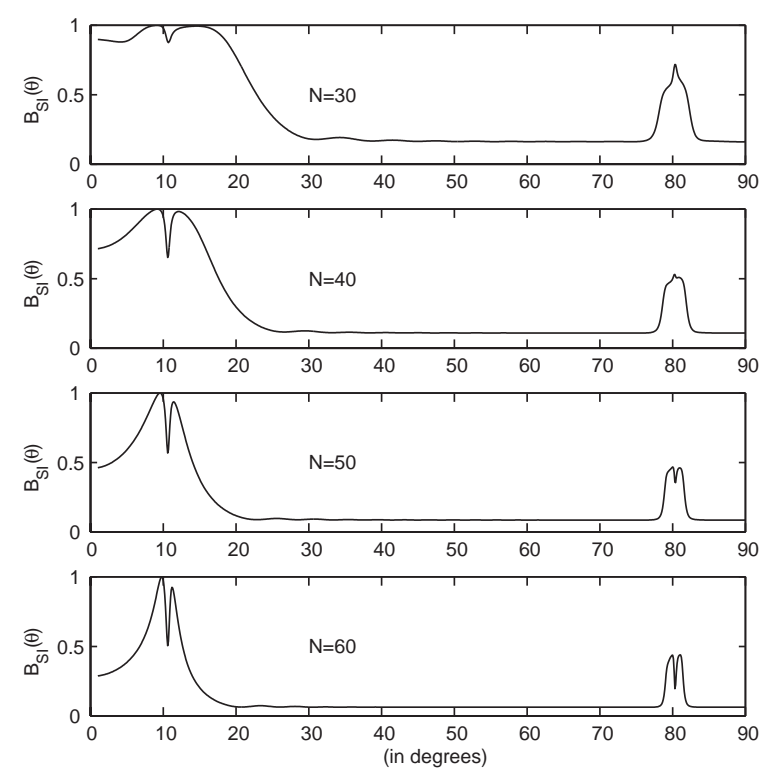

Fig. 12. Improvement in the resolution of SI processor with increasing $N$. Sources are at $10^{\circ}, 11^{\circ}, 80^{\circ}$ and $81^{\circ} . \mathrm{SNR}=0 \mathrm{~dB}$, $\Delta=\lambda / 2$.

comparable resolution at $10^{\circ}$. The advantage of increasing $N$ instead of increasing $\Delta$ is that ambiguity due to the possible appearance of large sidelobes is avoided. Especially at low SNR, increasing $N$ is a more reliable way of improving performance.

The effect of reduction in SNR on the performance of SI processor is depicted in Fig. 13. It is seen once again that decreasing SNR leads to a more rapid degradation of performance near the endfire direction.

The degradation in performance with decreasing SNR can be arrested by increasing the number of snapshots. The effect of increasing the number of snap- shots from 200 to 1000 is shown in Fig. 14. This figure shows plots of $B_{\mathrm{SI}}(\theta)$ for 2 sources at $10^{\circ}$ and $80^{\circ}$, and $0 \mathrm{~dB}$ SNR. The peak at $10^{\circ}$ is seen only in the panel corresponding to 1000 snapshots. The peak at $80^{\circ}$ is seen in all the panels, but the sharpness of this peak is seen to increase with an increase in the number of snapshots.

Extensive simulations have been carried out to compute the bias and mean square errors (MSE) of the estimated source directions as a function of SNR, number of sensors $N$, and the source direction $\theta$. A representative set of results is given in Figs. 15-17. 


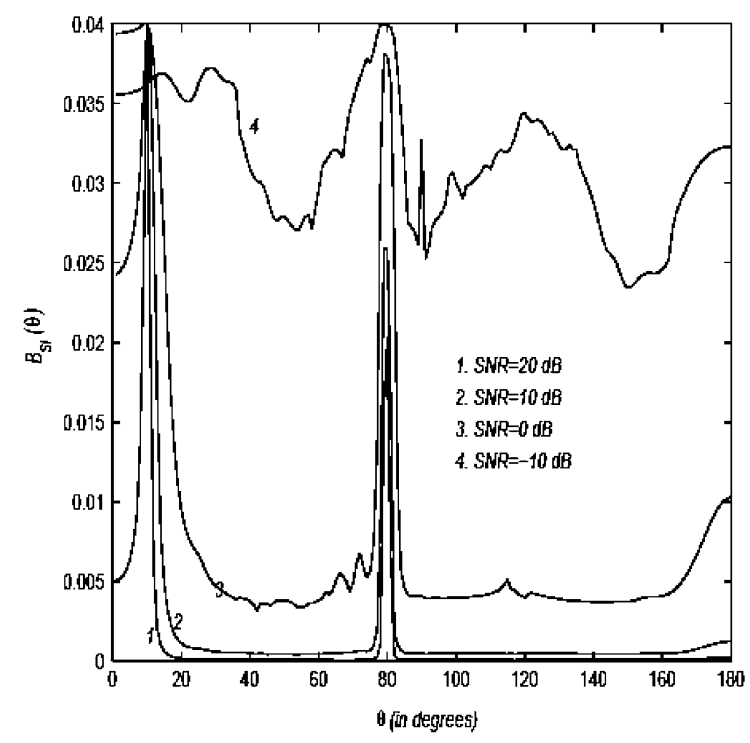

Fig. 13. Finite-data performance of the SI processor for different SNR. Two sources are present at $10^{\circ}, 80^{\circ} . f=50 \mathrm{~Hz}, N=30$, $\Delta=\lambda / 2$.

The bias and MSE were computed by averaging over 300 Monte Carlo simulations. The corresponding results for the MUSIC estimator are also given in these figures for comparison. While the MUSIC estimator performs better, it may be recalled that MUSIC requires either a 3-D search or a prior information of the source range and depth. Also, the MUSIC estimator requires the knowledge of all the ocean acoustic parameters while the SI algorithm needs only the knowledge of the modal wavenumbers.

Knowledge of the modal wavenumbers $\left\{k_{m}, m=\right.$ $1, \ldots, M\}$ is required for bearing estimation. Values of $k_{m}$ may be either computed from known/assumed values of the channel parameters or estimated from acoustic field data using a wavenumber estimation technique $[3,8,14]$. In either case, the assumed values of $k_{m}$ may not be the true values. Fig. 18 shows the effect of errors in the assumed values of $k_{m}$ on the SI processor response function $B_{\mathrm{SI}}(\theta)$. The received signal vector for a $50 \mathrm{~Hz}$ source in the Pekeris channel was computed using the actual wavenumbers $k_{m}$. But the modal steering vectors were computed assuming erroneous wavenumbers $\hat{k}_{m}=k_{m}(1+\varepsilon / 100)$, where the error percentage $\varepsilon$ is the same for all the modes. It is seen from Fig. 18 that the bearing esti-
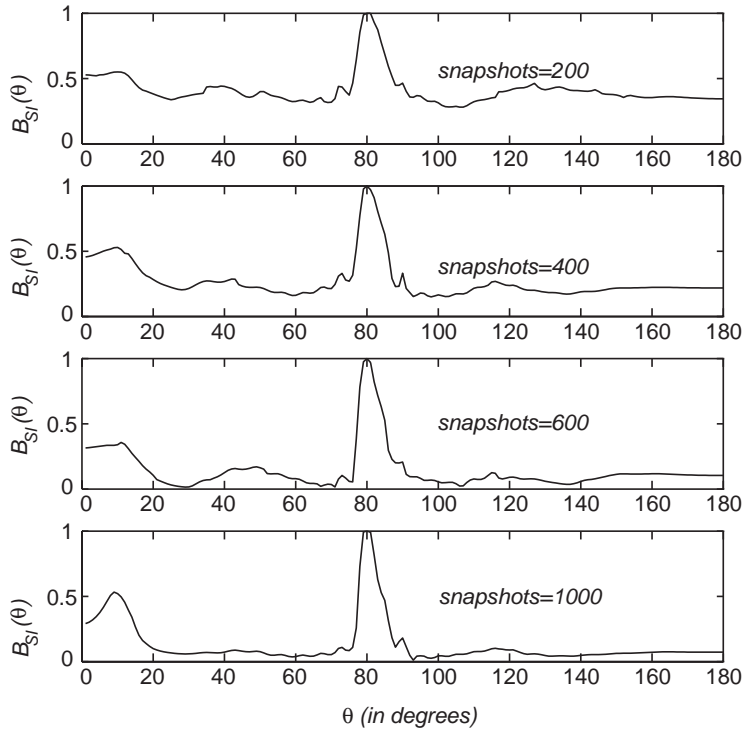

Fig. 14. Effect of variation of the number of snapshots on the finite-data performance of the SI processor at $0 \mathrm{~dB}$ SNR. Two sources are present at $10^{\circ}$ and $80^{\circ} . f=50 \mathrm{~Hz}, N=30, \Delta=\lambda / 2$.
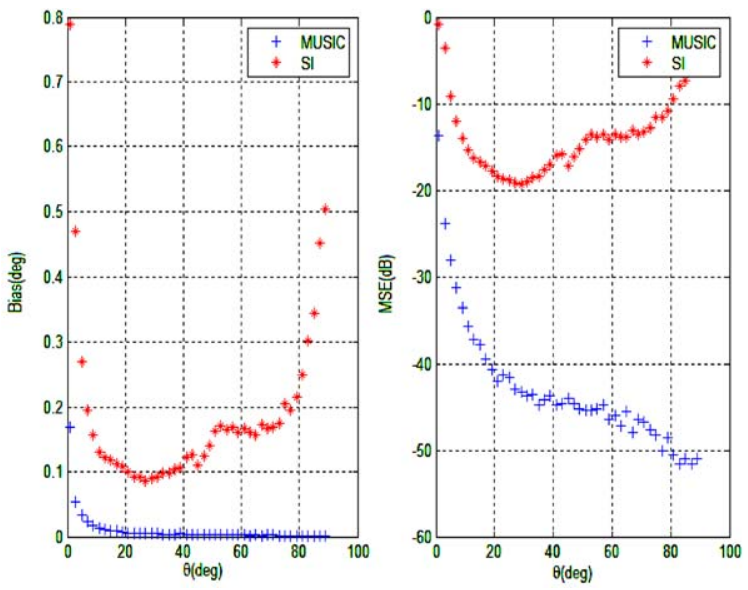

Fig. 15. Plots of the bias and MSE of the estimated source direction as a function of $\theta$ for fixed $N$ and SNR.

mates have a positive/negative bias according as the assumed wavenumbers have a positive/negative error. It is also seen that bearing estimates are not very sensitive to wavenumber errors for sources near broadside. Sensitivity to wavenumber mismatch increases as a source approaches the endfire direction. 

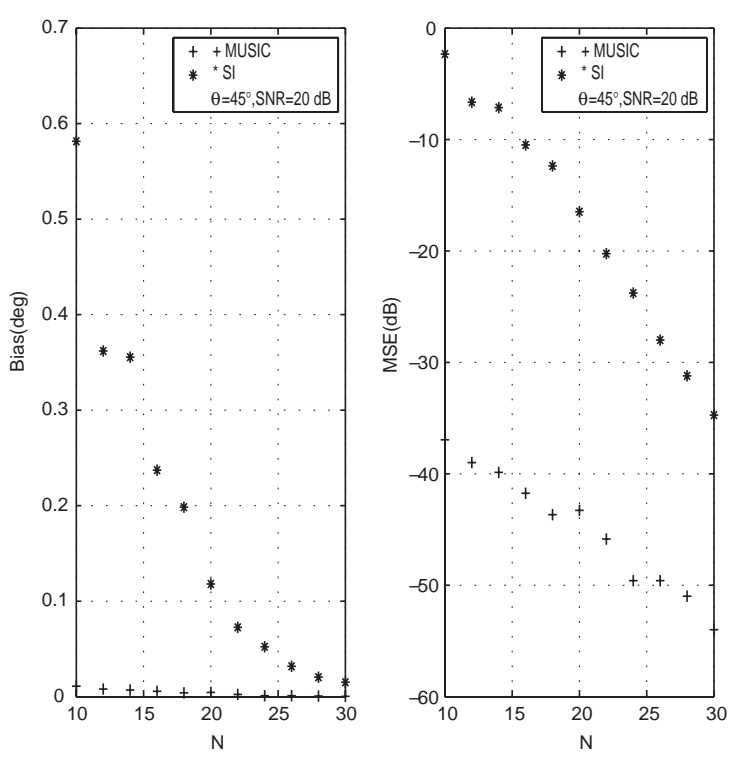

Fig. 16. Plots of the bias and MSE of the estimated source direction as a function of $N$ for fixed $\theta$ and SNR.
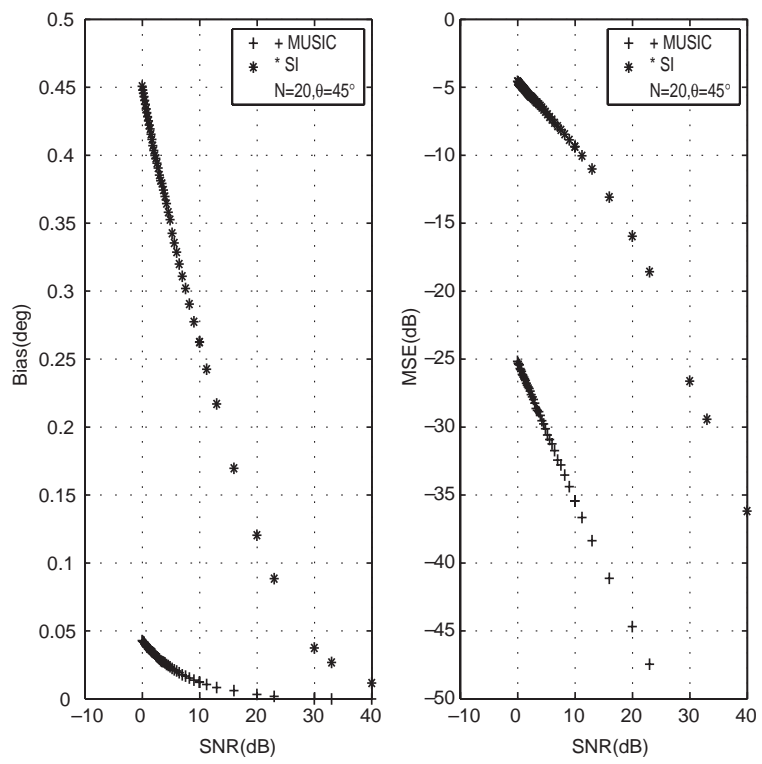

Fig. 17. Plot of the bias and MSE of the estimated source direction as a function of SNR for fixed $\theta$ and $N$.

\section{Summary and conclusions}

In the absence of prior knowledge of source range and depth, all known bearing estimation techniques
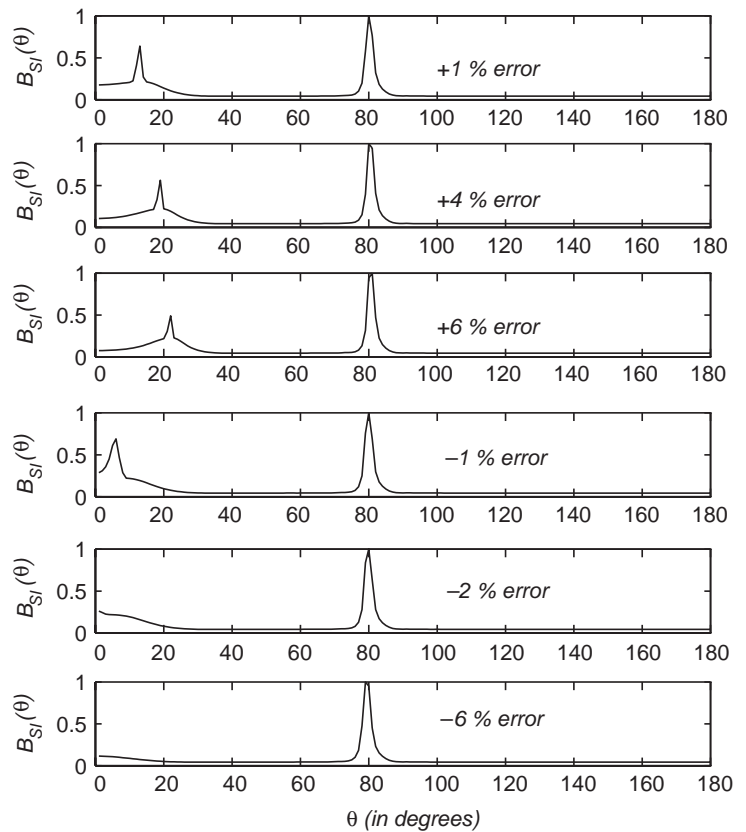

Fig. 18. Effect of errors in the assumed values of $k_{m}$ on the finite data performance of the SI processor for 2 sources at $10^{\circ}$ and $80^{\circ} . f=50 \mathrm{~Hz}, N=30, \Delta=\lambda / 2$.

invoke either a plane-wave assumption or make a simultaneous estimation of range and depth also. It has been shown that a plane-wave assumption leads to biased estimates, the bias being higher for sources closer to the endfire direction. Simultaneous estimation of bearing, range and depth using MFP involves a highly computation-intensive three-dimensional search. The subspace-intersection (SI) algorithm presented in this paper yields unbiased bearing estimates without requiring prior or concurrent range-depth estimation. Also, the environmental information (modal wavenumbers) required by the SI algorithm is less exhaustive and more easily obtainable than that required by MFP.

The SI algorithm exploits the relationship between the signal subspace and the modal subspace. In common with other subspace-based direction-ofarrival estimation algorithms, the SI algorithm possesses the attributes of high resolution and multiple-source bearing estimation capability. Prior knowledge of the number of sources is necessary for a reliable determination of the signal subspace. The 
SI method requires relatively large arrays, and the number of sensors required for satisfactory performance increases with an increase in the number of modes in the channel. Theoretically, $N \geqslant M(J+1)$ is a sufficient condition for unambiguous localization of $J$ sources while $N \geqslant M+J$ is a necessary condition, where $N$ is the number of sensors and $M$ is the number of modes. But under the limitation of finite data in a noisy channel, much larger values of $N$ may be required to increase the array gain for obtaining a satisfactory performance. The resolution of the SI algorithm is not uniform for all directions, the resolution being lower for sources closer to the endfire direction. This nonuniformity may be avoided by using a circular array [10]. The resolution of the SI algorithm can be enhanced either by increasing the number of sensors $N$ or by increasing the interelement spacing $\Delta$. But, if $\Delta>\lambda_{1} / 2$, appearance of large sidelobes may cause an ambiguity in localization.

In conclusion, the SI algorithm presented in this paper has several attractive features compared to all known methods of bearing estimation in a horizontally stratified ocean. Even when three-dimensional source localization is required it is often important to estimate bearing with a higher degree of accuracy than range and depth. It may then be preferable to use the SI method for bearing estimation and use MFP for range-depth estimation only, since the SI method is less vulnerable to environmental uncertainty than MFP.

\section{Acknowledgements}

The authors would like to acknowledge an anonymous reviewer for many useful suggestions and comments. This work was partially supported by the Naval Physical and Oceanographic Laboratory, Kochi, India.

\section{Appendix A. Linear independence of signal vectors}

Consider the signal vectors $\left\{\boldsymbol{p}\left(\boldsymbol{x}_{j}^{\prime}\right) ; j=1, \ldots, J+\right.$ $1\}$ at a uniform horizontal linear array generated by $(J+1)$ sources with arbitrary position vectors $\left\{\boldsymbol{x}_{j}^{\prime}=\right.$ $\left.\left(\theta_{j}^{\prime}, r_{j}^{\prime}, z_{j}^{\prime}\right) ; j=1, \ldots, J+1\right\}$ and distinct bearing angles $\left\{\theta_{j}^{\prime} ; j=1, \ldots, J+1\right\}$ in the interval $[0, \pi]$. Let the angles be ordered as

$\cos \theta_{1}^{\prime}>\cos \theta_{2}^{\prime}>\cdots>\cos \theta_{J+1}^{\prime}$.

We recall that

$\boldsymbol{p}\left(\boldsymbol{x}_{j}^{\prime}\right)=\sum_{m=1}^{M} b_{m j} \boldsymbol{a}\left(k_{m} \cos \theta_{j}^{\prime}\right), \quad j=1, \ldots, J+1$,

$$
\begin{aligned}
& \boldsymbol{a}\left(k_{m} \cos \theta_{j}^{\prime}\right)=\left[1 \mathrm{e}^{\mathrm{i} k_{m} \Delta \cos \theta_{j}^{\prime}} \cdots \mathrm{e}^{\mathrm{i}(N-1) k_{m} \Delta \cos \left(\theta_{j}^{\prime}\right)}\right]^{\mathrm{T}}, \\
& \quad m=1, \ldots, M \\
& k_{1}>k_{2}>\cdots>k_{M} .
\end{aligned}
$$

We shall prove that $\boldsymbol{p}\left(\boldsymbol{x}_{1}^{\prime}\right), \ldots, \boldsymbol{p}\left(\boldsymbol{x}_{J+1}^{\prime}\right)$ are linearly independent if the sensor array satisfies the following conditions:

$N \geqslant M(J+1)$,

$\Delta<\pi / k_{1}=\lambda_{1} / 2$

where $\lambda_{1}=2 \pi / k_{1}$ is the wavelength of the first normal mode.

Proof. We note that, in view of (A.1) and (A.4), we have for all $i \in\{1,2, \ldots, J+1\}$

$k_{1} \cos \theta_{i}^{\prime}>k_{m} \cos \theta_{i}^{\prime} \quad$ for $m=2, \ldots, M$

$k_{1} \cos \theta_{i}^{\prime}>k_{m} \cos \theta_{j}^{\prime} \quad$ for $m=1, \ldots, M$;

$$
j=i+1, \ldots, J+1 .
$$

Consider a linear combination of the signal vectors $\sum_{j=1}^{J+1} c_{j} \boldsymbol{p}\left(\boldsymbol{x}_{j}^{\prime}\right)$. We can write

$$
\begin{aligned}
\sum_{j=1}^{J+1} c_{1} \boldsymbol{p}\left(\boldsymbol{x}_{j}^{\prime}\right)= & c_{1} b_{11} \boldsymbol{a}\left(k_{1} \cos \theta_{1}^{\prime}\right) \\
& +c_{1} \sum_{m=2}^{M} b_{m 1} \boldsymbol{a}\left(k_{m} \cos \theta_{1}^{\prime}\right) \\
& +\sum_{m=1}^{M} \sum_{j=2}^{J+1} c_{j} b_{m j} \boldsymbol{a}\left(k_{m} \cos \theta_{j}^{\prime}\right) .
\end{aligned}
$$

Inequalities (A.5)-(A.7) imply that the vector $\boldsymbol{a}\left(k_{1} \cos \theta_{1}^{\prime}\right)$ is linearly independent of all the other vectors on the right-hand side of Eq. (A.8). Also, we 
shall assume that neither the source nor the sensor array is at the ocean surface. It follows that

$b_{1 j} \neq 0 \quad \forall j$,

since $b_{1 j}$ is the first mode amplitude of the $j$ th source signal, which can be zero only if either the source or the sensor array is on the oceanic surface. Hence,

$\sum_{j=1}^{J+1} c_{j} \boldsymbol{p}\left(\boldsymbol{x}_{j}^{\prime}\right)=0 \Rightarrow c_{1}=0 \Rightarrow \sum_{j=2}^{J+1} c_{j} \boldsymbol{p}\left(\boldsymbol{x}_{j}^{\prime}\right)=0$.

Proceeding in a similar fashion, we can show that $\sum_{j=2}^{J+1} c_{j} \boldsymbol{p}\left(\boldsymbol{x}_{j}^{\prime}\right)=0 \Rightarrow c_{2}=0 \Rightarrow \sum_{j=3}^{J+1} c_{j} \boldsymbol{p}\left(\boldsymbol{x}_{j}^{\prime}\right)=0$ and so on. It follows that $\sum_{j=1}^{J+1} c_{j} \boldsymbol{p}\left(\boldsymbol{x}_{j}^{\prime}\right)=0 \Rightarrow c_{1}=$ $c_{2}=\cdots=c_{J+1}=0$. Hence the vectors $\left\{\boldsymbol{p}\left(\boldsymbol{x}_{j}^{\prime}\right) ; j=\right.$ $1, \ldots, J+1\}$ are linearly independent.

\section{Appendix B. Determination of $Q(\theta)$ for large $N$}

The unit vector $\boldsymbol{e}(\theta)$ is defined as [see Eq. (57)]

$\boldsymbol{e}=\frac{\sum_{m=1}^{M} b_{m j} \boldsymbol{a}\left(k_{m} \cos \theta\right)}{\left\|\sum_{m=1}^{M} b_{m j} \boldsymbol{a}\left(k_{m} \cos \theta\right)\right\|_{2}}$

and

$$
\begin{aligned}
& \left\|\sum_{m=1}^{M} b_{m j} \boldsymbol{a}\left(k_{m} \cos \theta\right)\right\|_{2}^{2} \\
& \quad=\sum_{n=0}^{N-1}\left|\sum_{m=1}^{M} b_{m j} \exp \left(\mathrm{in} k_{m} \Delta \cos \theta\right)\right|^{2} \\
& =\sum_{n=0}^{N-1} \sum_{l=1}^{M} \sum_{m=1}^{M} b_{i j} b_{m j}^{*} \exp \left[\mathrm{in}\left(k_{l}-k_{m}\right) \Delta \cos \theta\right] .
\end{aligned}
$$

Denoting the magnitude and phase of $b_{m j}$ by $B_{m}$ and $\Phi_{m}$, respectively, we get

$$
\left\|\sum_{m=1}^{M} b_{m j} \boldsymbol{a}\left(k_{m} \cos \theta\right)\right\|_{2}^{2}
$$

$$
\begin{aligned}
= & \sum_{n=0}^{N-1} \sum_{l=1}^{M} \sum_{m=1}^{M} B_{l} B_{m} \exp \left[\mathrm{i}\left(\phi_{l}-\phi_{m}\right)\right] \\
& \exp \left[\mathrm{in}\left(k_{l}-k_{m}\right) \Delta \cos \theta\right] \\
= & N \sum_{m=1}^{M} B_{m}^{2}+2 \sum_{l=1}^{M} \sum_{m=1}^{M} B_{l} B_{m} \\
& \times\left\{\cos \left(\phi_{l}-\phi_{m}\right) \sum_{n=0}^{N-1} \cos \left[n\left(k_{l}-k_{m}\right) \Delta \cos \theta\right]\right. \\
& \left.-\sin \left(\phi_{l}-\phi_{m}\right) \sum_{n=0}^{N-1} \sin \left[n\left(k_{l}-k_{m}\right) \Delta \cos \theta\right]\right\} \\
\cong & N \sum_{m=1}^{M} B_{m}^{2} \quad \text { if } N \gg\left|\frac{2 \pi}{\left(k_{l}-k_{m}\right) \Delta \cos \theta}\right| . \quad \text { B } .3
\end{aligned}
$$

From Eqs. (B.1) and (B.3), we get

$$
\begin{aligned}
\left\|\frac{\mathrm{d} \boldsymbol{e}(\theta)}{\mathrm{d} \theta}\right\|_{2}^{2}= & \frac{1}{N \sum_{m=1}^{M} B_{m}^{2}} \\
& \times\left\|\frac{\mathrm{d}}{\mathrm{d} \theta} \sum_{m=1}^{M} b_{m j} \boldsymbol{a}\left(k_{m} \cos \theta\right)\right\|_{2}^{2} .
\end{aligned}
$$

Consider

$$
\begin{aligned}
\left\|\frac{\mathrm{d}}{\mathrm{d} \theta} \sum_{m=1}^{M} b_{m j} \boldsymbol{a}\left(k_{m} \cos \theta\right)\right\|_{2}^{2} \\
=\sum_{n=1}^{N-1}\left|\sum_{m=1}^{M} n b_{m j} k_{m} \Delta \sin \theta \exp \left(\mathrm{in} k_{m} \Delta \cos \theta\right)\right|^{2} \\
=\sum_{n=1}^{N-1} \sum_{l=1}^{M} \sum_{m=1}^{M} n^{2} B_{l} B_{m} k_{l} k_{m} \Delta^{2} \sin ^{2} \theta \\
\quad \times \exp \left[\mathrm{i}\left(\phi_{l}-\phi_{m}\right)\right] \exp \left[\mathrm{i} n\left(k_{l}-k_{m}\right) \Delta \cos \theta\right] \\
=\frac{N(N-1)(2 N-1)}{6} \sum_{m=1}^{M} B_{m}^{2} k_{m}^{2} \Delta^{2} \sin ^{2} \theta
\end{aligned}
$$




$$
\begin{aligned}
& +2 \Delta^{2} \sin ^{2} \theta \sum_{l=1}^{M} \sum_{m=1}^{M} B_{l} B_{m} k_{l} k_{m}\left\{\cos \left(\phi_{l}-\phi_{m}\right)\right. \\
& \times \sum_{n=1}^{N-1} n^{2} \cos \left[n\left(k_{l}-k_{m}\right) \Delta \cos \theta\right]+\sin \left(\phi_{l}-\phi_{m}\right) \\
& \left.\times \sum_{n=1}^{N-1} n^{2} \sin \left[n\left(k_{l}-k_{m}\right) \Delta \cos \theta\right]\right\} \\
& \cong \frac{1}{3} N^{3} \sum_{m=1}^{M} B_{m}^{2} k_{m}^{2} \Delta^{2} \sin ^{2} \theta \\
& \text { if } N \gg\left|\frac{2 \pi}{\left(k_{l}-k_{m}\right) \Delta \cos \theta}\right| .
\end{aligned}
$$

The last step in (B.5) follows from the fact that $\sum_{n=1}^{N} n^{2} \cos n x=O\left(N^{2}\right)$ and $\sum_{n=1}^{N} n^{2} \sin n x=O\left(N^{2}\right)$ if $N x \gg 2 \pi$. It follows from (B.4) and (B.5) that

$$
\begin{aligned}
Q(\theta) & =\left\|\frac{\mathrm{d}(\theta)}{\mathrm{d} \theta}\right\|_{2} \\
& \cong \frac{1}{\sqrt{3}} N \Delta\left\{\frac{\sum_{m=1}^{M} k_{m}^{2}\left|b_{m j}\right|^{2}}{\sum_{m=1}^{M}\left|b_{m j}\right|^{2}}\right\}^{1 / 2}|\sin \theta| .
\end{aligned}
$$

\section{References}

[1] L.M. Brekhovskikh, Yu. Lysanov, Fundamentals of Ocean Acoustics, Springer, Berlin, FRG, 1982 (Chapter 5).

[2] M.J. Buckingham, On the response of a towed array to the acoustic field in shallow water, IEE Proc. Pt-F 131 (1984) 298-307.

[3] H.M. Chouhan, G.V. Anand, Normal mode wave-number estimation using a towed array, J. Acoust. Soc. Amer. 93 (1993) 1807-1814
[4] G.H. Golub, C.F. Van Loan, Matrix Computations, Johns Hopkins University Press, Maryland, 1996.

[5] J. Ianniello, A MATLAB version of the KRAKEN normal mode code, Technical Memorandum, Naval Undersea Warfare Center Detatchment, New London C.T., 1994.

[6] C. Karthikeyan, Directional response of a towed array in shallow sea, IEE Proc. Pt-F 133 (1986) 138-145.

[7] R. Klemm, Low-error bearing estimation in shallow water, IEEE Trans. Aerospace Electronic Systems 18 (1982) $1340-1342$.

[8] L.G. Krasny, S.P. Antonyuk, Wave-number estimation in an ocean waveguide, J. Acoust. Soc. Amer. 102 (1997) 2697-2704.

[9] R. Kumaresan, D.W. Tufts, Estimating the angles of arrival of multiple plane waves, IEEE Trans. Aerospace Electronic Systems 19 (1983) 134-139.

[10] S. Lakshmipathi, Three dimensional localization of acoustic sources in the ocean, Ph.D. Thesis, Department of Electrical Communication Engineering, Indian Institute of Science, Bangalore, India, 1999.

[11] F.W. Machell, C.S. Perrod, G.E.Ellis, Stastical characteristics of ocean acoustic noise processes, in: E.J. Wegman, S.C. Schwartz, J.B. Thomas (Eds.), Topics in Non-Gaussian Signal Processing, Springer, New York, 1989, pp. 29-57 (Chapter $3)$.

[12] R. Roy, A. Paulraj, T. Kailath, ESPRIT -A subspace rotation approach to estimation of parameter of cisoids in noise, IEEE Trans. Acoust. Speech Signal Process. 34 (1986) $1340-1342$.

[13] R.O. Schmidt, Multiple emitter location and signal parameter estimation, Proceedings of RADC Spectrum Estimation Workshop, Rome Air Development Center, Rome, 1979, pp. 243-258.

[14] E.C. Shang, H.P. Wang, Z.Y. Huang, Waveguide characterisation and source localisation in shallow water using Prony method, J. Acoust. Soc. Amer. 83 (1988) 103-108.

[15] P. Stoica, A. Nehorai, MUSIC, maximum likelihood and Cramer-Rao bound, IEEE Trans. Acoust. Speech Signal Process. 37 (1989) 720-740.

[16] A. Tolstoy, Matched Field Processing for Underwater Acoustics, World Scientific, Singapore, 1993. 